\title{
Scale Transformations in Metric-Affine Geometry
}

\author{
Damianos Iosifidis ${ }^{1}(\mathbb{D}$ and Tomi Koivisto $2,3,4,5, *$ (D) \\ 1 Institute of Theoretical Physics, Department of Physics, Aristotle University of Thessaloniki, \\ 54124 Thessaloniki, Greece; diosifid@auth.gr \\ 2 Nordita, KTH Royal Institute of Technology and Stockholm University, Roslagstullsbacken 23, \\ SE-10691 Stockholm, Sweden \\ 3 Laboratory of Theoretical Physics, Institute of Physics, University of Tartu, W. Ostwaldi 1, \\ 50411 Tartu, Estonia \\ 4 National Institute of Chemical Physics and Biophysics, Rävala pst. 10, 10143 Tallinn, Estonia \\ 5 Helsinki Institute of Physics and Department of Physical Sciences, Helsinki University, P.O. Box 64, \\ FIN-00014 Helsinki, Finland \\ * Correspondence: tomi.koivisto@nordita.org or tomik@astro.uio.no
}

Received: 17 January 2019; Accepted: 11 March 2019; Published: 15 March 2019

\begin{abstract}
This article presents an exhaustive classification of metric-affine theories according to their scale symmetries. First it is clarified that there are three relevant definitions of a scale transformation. These correspond to a projective transformation of the connection, a rescaling of the orthonormal frame, and a combination of the two. The most general second order quadratic metric-affine action, including the parity-violating terms, is constructed in each of the three cases. The results can be straightforwardly generalised by including higher derivatives, and implemented in the general metric-affine, teleparallel, and symmetric teleparallel geometries.
\end{abstract}

Keywords: scale invariance; Palatini formalism; metric affine gauge theory of gravity

\section{Introduction}

The concept of gauge transformation was introduced into physics a century ago in the work of Hermann Weyl [1], who generalised the spacetime geometry in Einstein's recent General Theory of Relativity (GR) by incorporating the relativity of magnitudes. The (pseudo-)Riemannian geometry of GR describes a curved spacetime, wherein the direction of a vector parallel transported around a closed loop can rotate. In Weyl geometry, the magnitude of the vector can also change. The theory was made to be invariant under the simultaneous transformation of the gravitational metric and the electromagnetic field, which was the basis for a geometrical unification of the two fundamental interactions known at the time.

About a decade later it was clarified that the electromagnetic interaction requires rather the compact gauge group $U(1)$ [2], and the extension of the gauge principle to non-Abelian groups has eventually lead to the great successes of the modern standard model of particle physics that begun some fifty years ago [3]. Thus, the very foundation of current theoretical physics can be traced back to the geometrical analysis of GR, and at its the core lie the issues of scales and integrability [4]. As expressed in Reference [5]: "It was Weyl's desire to remove all elements of an action at a distance theory from geometry. The direction of a vector in Riemannian geometry became nonintegrable, but its length remains integrable. This situation Weyl wanted to change. Weyl's interpretation of the Weyl covector $Q$ as electromagnetic potential turned out not to be viable-basically because the electric charge has no intrinsic relation to the geometry of spacetime-but the geometry Weyl created will reappear as linked to the gauge theory of scale transformations". Currently, there is still no consensus on the status of scale invariance in physics, but a natural viewpoint is to regard it as a fundamental, 
exact symmetry which is broken in a spontaneous fashion [6]. For reviews on various aspects of scale invariance, see [7-11].

The aim of this paper is to study scale invariance and closely related symmetries in the general geometrical framework of metric-affine gravity [7]. We work mostly in the so called Palatini formalism, where the independent gravitational variables are the metric and the affine connection $[12,13]$. As well-known, this formalism extends the (pseudo-)Riemannian framework by taking into account two fundamental properties of spacetime geometry besides the (metric) curvature: torsion $[14,15]$ and non-metricity $[4,16]$, the former dating back to the work Cartan, the prototype manifestation of the latter being the Weyl covector $Q$. While in the first formulation of GR the metric was the only variable and gravitation was attributed to its curvature, Einstein himself a few years later considered an alternative formulation in terms of torsion, and in general became to uphold the view that the spacetime connection, rather than the metric, should be regarded as the "directly relevant conceptual element" [5]. In a spacetime equipped with an independent connection, new possibilities arise to realise the transformation of physical scales. As Weyl had clarified, the connection may be retained invariant while the metric is transformed [17]. On the other hand, the projective transformation a.k.a. the $\lambda$-transformation, which is a change of the connection that leaves its autoparallels invariant, was a recurrent theme in the early history of unified field theory [18], and it indeed at the same time it is related to the gauge transformation of the electromagnetic field and the geometric construction of the famous Ehlers-Pirani-Schild method, which is basically the separation of the conformal and the projective structures on a manifold [19].

A specific task we are about to undertake is the systematic classification of quadratic metric-affine theories according to their rescaling and projective symmetries. Currently, there is a resurgence of activity in alternative formulations of gravity theory in the context generalised geometry, especially motivated by the mysteries of modern cosmology [13]. The most extensively studied example is the $f(R)$ gravity, wherein the important role of the conformal relation has been eludicated in the metric models [20,21], the Palatini models [22,23] and their unifications [24,25]. In a more generic context, the interest of many investigators is captured by "the trinity of gravity" [13] i.e., the facts that GR can be equivalently formulated in terms of either curvature, torsion or non-metricity, and that furthermore, each of these three formulations may give rise to inequivalent generalisations, thus opening new avenues to address the cosmological problems and the more long-standing foundational issues in the theory of gravity. In this paper we also pave the way for explorations into the general teleparallel geometry that is flat but exhibits both non-metricity and torsion. Recently, interesting insights have been found into a possible duality between the latter two, mediated via the projective transformation $[23,26,27]$. The many possible applications of scale-invariant theories include unification [1,4], the hierarchy problem [28,29], the cosmological constant problem [30,31], dark energy [32-34], dark matter [8,35], leptogenesis [36], wormholes [37,38], non-singular black holes [39,40] and their evaporation [41,42], the origin of time [43], inflation [44,45] and its bouncing [46] and other [47] alternatives. Previously various versions of scale invariance in metric-affine geometry have been considered in e.g., References [7,48-56], and some recent discussions of projective invariance in metric-affine geometry are found in References [23,26,27,57-59].

Weyl's original theory [1] was written in terms of the unique scale-invariant quadratic Riemannian curvature scalar constructed from the metric and the metrical connection. It is well-known that this term, the "Weyl tensor squared", is higher order in derivatives, and in fact accommodates a ghost. Dirac had already discarded this particular formulation of the action principle on aesthetic grounds, though he recognised Weyl's geometric unification as "the outstanding one, unrivalled by its simplicity and beauty" [60]. Dirac nevertheless continued to work within the framework of (extended) metrical Riemannian geometry. His resolution was to introduce a compensating field, $\psi$, which has the 
transformation law $\psi \rightarrow e^{-\phi} \psi$ under the rescaling $g_{\mu \nu} \rightarrow e^{2 \phi} g_{\mu v}$ of the metric ${ }^{1} g_{\mu v}$, and couple this field non-minimally to the Einstein-Hilbert term. With a particular coefficient of the kinetic term for the field $\psi$, the non-minimally coupled scalar field action becomes locally scale-invariant. Presently, the $\psi$ is often known as the dilaton, and the action of the Weyl-Dirac theory (see [61] for an introduction) is referred to as the conformally coupled Brans-Dicke scalar-tensor theory. Here we shall follow the Dirac's approach and generalise the theory into metric-affine geometry. The task is to deduce the "co-covariant" terms that can be constructed from torsion and non-metricity. The first step is to catalogue all the diffeomorphism-invariant terms and the step that will follow is the construction of their scale-covariant combinations.

We have made an attempt at a systematic and pedagogic presentation of this paper. It is therefore organised such that many detailed derivations are included in the three Appendices, which also contain some simple illustrative examples. There is often some confusion about the definitions and especially the physical interpretations of the scale transformations, an example being the errors that regularly arise about the (non)equivalence of the Jordan and the Einstein frames in the $f(R)$ and other scalar-tensor theories [62], despite that the issue was explained in exemplary clarity already in the seminal paper of Brans and Dicke [63]. For these reasons, we discuss the basics of a scale-invariant theory at some length. First, in Section 2, we elaborate on the meaningful definitions of a scale transformation, from both the perspectives of spacetime geometry (greek indices in our notation) and tangent space geometry (latin indices), and then, in Section 3 we introduce a very simple example of scale invariant theory, the "Einstein-Cartan-Weyl-Dirac" action, in order to illustrate the formulation of such a theory and its physical interpretation. The main derivations and results of this paper are given in Sections 4 and 5, wherein we deduce the generic quadratic scale-covariant theories in terms of torsion and non-metricity in the parity-symmetric and -asymmetric cases, respectively. The results will be summarised in Table 2. Finally, we conclude in Section 6 by gathering the bunches of scalars in Table 3.

\section{The Geometrical Framework}

In this section, we will define the scale transformations to be considered in the following. We consider two formulations, which are referred to as the Palatini formalism and the metric-affine gauge formalism, for reasons explained in Reference [64].

\subsection{Scale Transformations in the Palatini Formalism}

In GR, the metric $g_{\mu v}$ is the only independent variable. The rescaling of the metric, by a coordinate-dependent function $\phi(x)$, is defined simply as

$$
g_{\mu \nu} \rightarrow e^{2 \phi} g_{\mu \nu} .
$$

The metric connection is given by the Christoffel symbol, the unique symmetric metric-compatible connection

$$
\left\{\begin{array}{c}
\alpha \\
\beta \gamma
\end{array}\right\}=\frac{1}{2} g^{\alpha \lambda}\left(g_{\beta \lambda, \gamma}+g_{\lambda \gamma, \beta}-g_{\beta \gamma, \lambda}\right) .
$$

Under the transformation Equation (1), this obviously transforms as

$$
\left.\left\{\begin{array}{c}
\alpha \\
\beta \gamma
\end{array}\right\} \rightarrow\left\{\begin{array}{c}
\alpha \\
\beta \gamma
\end{array}\right\}+\phi^{\alpha} g_{\beta \lambda}-2 \delta_{(\beta}^{\alpha} \phi, \lambda\right) .
$$

\footnotetext{
1 As is a usual practice, we refer to tensors by their components. Thus, e.g., the Weyl (co)vector $Q=Q_{\mu} \mathrm{d} x^{\mu}$ will be referred to
} as $Q_{\mu}$ from now on. 
This was the original form of the scaling transformation in Weyl's 1918 theory [1], where it was to be performed in concert with the gauge transformation of the electromagnetic field. One may consider the three new linear terms in the connection Equation (3) to appear with independent (constant) coefficients $w_{1}, w_{2}, w_{3}$,

$$
\left\{\begin{array}{c}
\alpha \\
\beta \gamma
\end{array}\right\} \rightarrow\left\{\begin{array}{c}
\alpha \\
\beta \gamma
\end{array}\right\}+w_{1} \phi^{\prime \alpha} g_{\beta \gamma}+w_{2} \delta_{\beta}^{\alpha} \phi, \gamma+w_{3} \delta_{\gamma}^{\alpha} \phi, \beta
$$

This generalisation of Weyl geometry can be called the linear vector distortion (where in the above, the vector field is reduced to the gradient of the scalar $\phi$ ), and it has been recently used to parameterise deviations from Riemannian geometry [26,65,66], see also [51,67-69].

In the paper at hand however, rather than employing a phenomenological parameterisation of the connection, we work in the Palatini formalism, wherein in addition to the metric, the affine connection $\Gamma_{\mu \nu}^{\alpha}$ is an independent dynamical variable [12]. The action for the gravitational theory is written in terms of both $g_{\mu \nu}$ and $\Gamma^{\alpha}{ }_{\mu v}$, and varied with respect to both of them independently. In this sense, the connection is not fixed a priori, but assumes its form dynamically, as a solution to its equation of motion. In such a theory, one reasonable realisation of the rescaling is simply $w_{1}=w_{2}=w_{3}=w_{4}=0$ [17], wherein

$$
\Gamma_{\beta \lambda}^{\alpha} \rightarrow \Gamma_{\beta \lambda}^{\alpha}+w_{1} \phi^{\alpha} g_{\beta \gamma}+w_{2} \delta_{\beta}^{\alpha} \phi_{, \gamma}+w_{3} \delta_{\gamma}^{\alpha} \phi_{, \beta}+w_{4} \epsilon_{\beta \gamma}^{\alpha \delta} \phi_{, \delta}
$$

would be the most general linear additive transformation. The different cases considered in this paper are summarised in Table 1.

Table 1. The projections of the affine connection Equation (4), or Equation (5), in terms of the vector distortion parameters in generalised Weyl geometry $[26,66]$.

\begin{tabular}{ccccc}
\hline Transformation & $w_{\mathbf{1}}$ & $w_{\mathbf{2}}$ & $\boldsymbol{w}_{\mathbf{3}}$ & Geometry \\
\hline conformal metric & $-\frac{1}{2}$ & $\frac{1}{2}$ & $\frac{1}{2}$ & Riemannian \\
conformal MAG & 0 & 0 & 0 & orthonormal \\
projective & 0 & 1 & 0 & holonomic \\
projective $/ /^{\prime}$ & 0 & $\frac{1}{2}$ & $-\frac{1}{2}$ & teleparallel \\
projective $_{\|}$ & 0 & $\frac{1}{2}$ & $\frac{1}{2}$ & symmetric telep. \\
\hline
\end{tabular}

Generically, the independent connection may have the metric-compatible piece Equation (2), but also non-metricity and torsion which do not necessarily reduce to pure vector or scalar components. The conventions we shall use are such that for the covariant derivative $\nabla_{\mu}$ of a vector $u^{\mu}$ with respect to the independent connection $\Gamma^{\alpha}{ }_{\mu v}$, we have

$$
\nabla_{\mu} u^{\lambda}=\partial_{\mu} u^{\lambda}+\Gamma^{\lambda}{ }_{\nu \mu} u^{v} .
$$

The curvature of the connection is defined as

$$
R_{\beta \mu \nu}^{\alpha}=2 \partial_{[\mu} \Gamma_{|\beta| v]}^{\alpha}+2 \Gamma_{\lambda[\mu}^{\alpha} \Gamma_{|\beta| v]}^{\lambda}
$$

the torsion $S_{\mu \nu}{ }^{\lambda}$ of the connection is given as its antisymmetric part,

$$
S_{\mu \nu}^{\lambda}=2 \Gamma^{\lambda}{ }_{[\mu \nu]}
$$

and the non-metricity $Q_{\alpha \mu v}$ as the covariant derivative of the metric,

$$
Q_{\alpha \mu \nu}=-\nabla_{\alpha} g_{\mu v} .
$$


One should be aware that various other conventions are used in the literature ${ }^{2}$. Clearly, in $n$ dimensions the torsion tensor can have $n^{2}(n-1) / 2$ and the non-metricity tensor $n(n+1) / 2$ independent components, which together comprise the $n^{3}$ independent components of a fully generic affine connection.

The geometric concepts such of angles, distances, areas and volumes are given by the metric. The special property of the Weyl rescaling Equation (5) is that it leaves angles invariant, whereas it changes the quantities involving magnitudes, i.e., lengths and its higher-dimensional generalisations. A priori, the geometric concept of parallel transport, of moving from one spacetime point to another, is completely independent of the metric. This is the compelling reason to adopt Palatini formalism, wherein the parallel transport is determined by the independent field $\Gamma^{\alpha}{ }_{\mu \nu}$. In particular, the geodesic equation that defines the parallel transport of a vector $u^{\mu}$ is $u^{\mu} \nabla_{\mu} u^{\alpha}=0$. There is an equivalence class of connections which describe the same geodesic paths. Any connection in this class is obtained from another by the projective transformation, which can be given by the one-form $\xi_{\mu}$ as

$$
\Gamma_{\mu \nu}^{\lambda} \rightarrow \Gamma_{\mu \nu}^{\lambda}+\delta_{\mu}^{\lambda} \xi_{v} .
$$

In analogy with the fact that rescaling of magnitudes has a priori nothing to do with the connection, the projective transformation has a priori nothing to do with the metric. It depends completely on the geometric prescription of the physical theory, whether they are related and what the relation would be. When the one-form above is a gradient, $\xi_{\mu}=\phi, \mu$, we obtain the special case of the linear vector distortion transformation Equation (4) with $w_{2}=1, w_{1}=w_{3}=0$. This has precisely the form of the electromagnetic gauge transformation [18,59] (up to the imaginary factor [4]). This suggests a special geometric relevance for this behaviour of the connection under rescalings, and indeed such will be clarified below in Section 2.2.

To summarise, we have arrived at a possible relation between the transformation Equation (10) for the connection and the rescaling Equation (1) for the metric, the two independent geometric objects of metric-affine geometry (or, Palatini formalism). We shall discard the relation Equation (3) from further consideration, since this relation was justified by the restriction to Riemannian geometry, which is ad hoc from our perspective, and besides has been already extensively studied in the past hundred years. Instead, we shall explore the transformations of metric-affine geometry, taking into account systematically all the three logical possibilities, as follows.

- Only the connection is transformed. This is called the projective transformation:

$$
\hat{\Gamma}_{\mu \nu}^{\lambda}=\Gamma_{\mu \nu}^{\lambda}+\delta_{\mu}^{\lambda} \xi_{v}, \quad \hat{g}_{\mu v}=g_{\mu v} .
$$

- Only the metric is transformed. This is called the conformal transformation:

$$
\bar{\Gamma}_{\mu \nu}^{\lambda}=\Gamma_{\mu \nu}^{\lambda}, \quad \bar{g}_{\mu \nu}=e^{2 \phi} g_{\mu \nu} .
$$

- Both the metric and the connection are transformed. This is called the frame rescaling:

$$
\tilde{\Gamma}_{\mu \nu}^{\lambda}=\Gamma_{\mu \nu}^{\lambda}+\delta_{\mu}^{\lambda} \partial_{\nu} \phi, \quad \tilde{g}_{\mu \nu}=e^{2 \phi} g_{\mu \nu} .
$$

All of them can be interpreted as scale transformations, or, "calibrations". As above, we shall denote the transformed quantities in the three cases with a hat (proper calibration), with a bar (orthonormal calibration) and with a tilde (holonomic calibration), respectively. The nomenclature can be explained better, and the physical interpretation of the transformations can be further elaborated,

2 For example, in the conventions of [64,70], the four previous equations would read $\nabla_{\mu} u^{\lambda}=\partial_{\mu} u^{\lambda}+\Gamma^{\lambda}{ }_{\mu \nu} u^{\nu}$, $R_{\beta \mu \nu}^{\alpha}=2 \partial_{[\mu} \Gamma_{\nu] \beta}^{\alpha}+2 \Gamma_{[\mu|\lambda|}^{\alpha} \Gamma_{v] \beta^{\lambda}}, T^{\lambda}{ }_{\mu \nu}=2 \Gamma_{[\mu v]}^{\lambda}$ and $Q_{\alpha \mu \nu}=\nabla_{\alpha} g_{\mu v}$, respectively. 
with the help of a further set of indices. For this purpose only we'll make a very brief excursion into the tangent space in the following subsection, which the reader may choose to skip.

\subsection{Scale Transformation in Metric-Affine Gauge Geometry}

To consider the underlying symmetry transformations in gravity theory it is useful to introduce a local basis $\partial_{a}{ }^{\mu}$, called the frame field, or the vielbein, which is a set of $n$ vector fields. The coframe field $\mathrm{e}^{a}{ }_{\mu}$ is the dual set of covectors which satisfy $\mathrm{e}^{a}{ }_{\nu} \partial_{a}{ }^{\mu}=\delta_{v}^{\mu}$, and $\mathrm{e}^{a}{ }_{\mu} \partial_{b}{ }^{\mu}=\delta_{b}^{a}$. We have the metric $\eta_{a b}$ which is convenient to consider in the Minkowski form. In terms of the frame field, the spacetime metric is given as

$$
g_{\mu \nu}=\eta_{a b} \mathrm{e}^{a}{ }_{\mu} \mathrm{e}^{b}{ }_{\nu}
$$

A fundamental field is then the connection one-form $\alpha^{a}{ }_{b \mu}$, and we denote covariant derivative the associated with this connection by $D_{\mu}$. The spacetime affine connection coefficients are given as

$$
\Gamma^{\alpha}{ }_{\mu \nu}=\partial_{a}{ }^{\alpha} D_{\mu} \mathrm{e}^{a}{ }_{\nu}=-\mathrm{e}^{a}{ }_{\nu} D_{\mu} \partial_{a}{ }^{\alpha} .
$$

This definition is often called "the tetrad postulate". Explicitly, the covariant derivative involves the connection $\alpha^{a}{ }_{b \mu}$ as,

$$
\begin{aligned}
& D_{\mu} \mathrm{e}^{a}{ }_{\nu}=\mathrm{e}^{a}{ }_{\nu, \mu}+\alpha^{a}{ }_{b \mu} \mathrm{e}^{b}{ }_{\nu} \\
& D_{\mu} \partial_{a}{ }^{v}=\partial_{a}{ }^{v}{ }_{, \mu}+\alpha_{a}{ }^{b}{ }_{\mu} \partial_{b}{ }^{v}
\end{aligned}
$$

and therefore the interpretation of the "tetrad postulate" is that the vielbein constant in the sense that it is preserved by the differentiation with respect to the sum of the two connections.

A general linear transformation is parameterised by $\Lambda^{a}{ }_{b}$, which has an inverse $\Lambda^{a}{ }_{c}\left(\Lambda^{-1}\right)^{c}{ }_{b}=\left(\Lambda^{-1}\right)^{a}{ }_{c} \Lambda^{c}{ }_{b}=\delta_{b}^{a}$, and its action in the orthonormal geometry is

$$
\begin{aligned}
\mathrm{e}^{a}{ }_{\mu} & \rightarrow \Lambda^{a}{ }_{b} \mathrm{e}^{b}{ }_{\mu} \\
\partial_{a}{ }^{\mu} & \rightarrow\left(\Lambda^{-1}\right)^{b}{ }_{a \partial_{b}}{ }^{\mu} \\
\alpha^{a}{ }_{b \mu} & \rightarrow \Lambda^{a}{ }_{c}\left(\alpha^{c}{ }_{d \mu}-\delta_{d}^{c} \partial_{\mu}\right)\left(\Lambda^{-1}\right)_{b}^{d} \\
\eta_{a b} & \rightarrow \eta_{a b} .
\end{aligned}
$$

The trace of the general linear transformation ${ }^{3}$ can parameterised by one parameter $\phi$ as $\bar{\Lambda}_{b}^{a}=e^{\phi} \delta_{b}^{a}$. Explicitly, we have then

$$
\begin{aligned}
\overline{\mathrm{e}}^{a}{ }_{\mu} & =e^{\phi} \mathrm{e}^{a}{ }_{\mu} \\
\bar{\partial}_{a}{ }^{\mu} & =e^{-\phi} \partial_{a}{ }^{\mu} \\
\bar{\alpha}^{a}{ }_{b \mu} & =\alpha_{b \mu}^{a}+\phi_{, \mu} \delta_{b}^{a} \\
\bar{\eta}_{a b} & =\eta_{a b}
\end{aligned}
$$

which gives a gauge transformation of the spin connection. From Equations (14) and (15), we have then

$$
\begin{aligned}
\bar{g}_{\mu \nu} & =e^{2 \phi} g_{\mu \nu} \\
\bar{\Gamma}_{\mu \beta}^{\alpha} & =\Gamma_{\mu \beta}^{\alpha} .
\end{aligned}
$$

3 In this paper we focus on scale invariance, but one could generalise these considerations to more general transformations. However, a theory that would be postulated to be invariant under a completely general transformation such as Equation (18), with no restrictions on $\Lambda_{b}^{a}$, would have to be topological in the sense of being invariant under arbitrary transformations of the metric. 
Therefore, the trace of the general linear transformation in orthonormal geometry is manifested in spacetime geometry as what we call the conformal transformation Equation (12) in this paper.

We can also consider a mere rescaling of the frame field, such that $\hat{\mathrm{e}}^{a}{ }_{\mu}=e^{\phi} \mathrm{e}^{a}{ }_{\mu}, \hat{\alpha}^{a}{ }_{b \mu}=\alpha^{a}{ }_{b \mu}$, which in view of Equations (14) and (15) implies that

$$
\begin{aligned}
\tilde{g}_{\mu v} & =e^{2 \phi} g_{\mu v} \\
\tilde{\Gamma}_{\mu \beta}^{\alpha} & =\Gamma^{\alpha}{ }_{\mu \beta}+\phi_{, \mu} \delta_{\beta}^{\alpha} .
\end{aligned}
$$

The latter transformation is a special case $\lambda_{\mu}=\phi_{, \mu}$ of what Einstein and others called the " $\lambda$-transformation" [18]

$$
\hat{\Gamma}^{\alpha}{ }_{\mu \beta} \rightarrow \Gamma^{\alpha}{ }_{\mu \beta}+\lambda_{\mu} \delta_{\beta}^{\alpha}
$$

This transformation amounts to a reparameterisation of the geodesic parameters, and thus leaves the autoparallels of the connection invariant. The case is of course the opposite to the frame rescaling in the sense that we obtain it by considering that the tangent space connection transforms as $\alpha_{b \mu}^{a} \rightarrow \Lambda_{c}^{a}\left(\alpha_{d \mu}^{c}-\delta_{d}^{c} \partial_{\mu}\right)\left(\Lambda^{-1}\right)_{b}^{d}$, but that the frame does not transform, $\mathrm{e}^{a}{ }_{\mu} \rightarrow \mathrm{e}^{a}{ }_{\mu}$. Clearly, for these reasons we can regard the combined effect Equations (22) and (23) in spacetime as a rescaling of the frame and the shift Equation (24) of the spacetime affinity as the projection of the connection in the tangent space.

In the orthonormal geometry we have considered up to this point, the tangent space can be seen to be defined by the constancy of the algebraic object $\eta_{a b}$. There is however another possible viewpoint. In a holonomic prescription, we rather regard the frame field as an absolute invariant. Then, it is not changed by any transformation, but rather transformations are understood to occur with respect to the frame field. The price to pay is then, obviously, that $\eta_{a b}$ has to be allowed to become "relative". In this prescription, a general linear transformation is

$$
\begin{aligned}
\mathrm{e}^{a}{ }_{\mu} & \rightarrow \mathrm{e}^{b}{ }_{\mu} \\
\partial_{a}{ }^{\mu} & \rightarrow \partial_{b}^{\mu} \\
\alpha_{b \mu}^{a} & \rightarrow \Lambda_{c}^{a}\left(\alpha_{d \mu}^{c}-\delta_{d}^{c} \partial_{\mu}\right)\left(\Lambda^{-1}\right)_{b}^{d} \\
\eta_{a b} & \rightarrow \eta_{c d}\left(\Lambda^{-1}\right)^{c}{ }_{a}\left(\Lambda^{-1}\right)_{b}^{d} .
\end{aligned}
$$

Now the trace of the general transformation, if parameterised as $\tilde{\Lambda}_{b}^{a}=e^{-\phi} \delta_{b}^{a}$, results in $\tilde{\eta}_{a b}=e^{2 \phi} \eta_{a b}$ and thus, from Equation (14) in that $\tilde{g}_{\mu v}=e^{2 \phi} g_{\mu v}$, and from Equation (15) we readily obtain the same result as in Equation (23). Thus, the trace of the linear transformation in holonomic geometry can be interpreted as a frame rescaling in orthogonal geometry. Alternatively we could refer to Equation (12) as the "orthogonal calibration" and to Equation (13) as the "holonomic calibration".

Finally, the projective transformation Equation (11) could be called the "proper calibration". The spacetime metric Equation (14) is obviously left invariant if we transform both the frame and the metric of the tangent space, i.e., consider the general linear transformation of the tangent space variables

$$
\begin{aligned}
\mathrm{e}^{a}{ }_{\mu} & \rightarrow \Lambda^{a}{ }_{b} \mathrm{e}^{b}{ }_{\mu} \\
\partial_{a}{ }^{\mu} & \rightarrow\left(\Lambda^{-1}\right)^{b}{ }_{a{ }_{b}}{ }^{\mu} \\
\alpha^{a}{ }_{b \mu} & \rightarrow \Lambda^{a}{ }_{c}\left(\alpha^{c}{ }_{d \mu}-\delta_{d}^{c} \partial_{\mu}\right)\left(\Lambda^{-1}\right)_{b}{ }_{b} \\
\eta_{a b} & \rightarrow \eta_{c d}\left(\Lambda^{-1}\right)^{c}{ }_{a}\left(\Lambda^{-1}\right)_{b}^{d} .
\end{aligned}
$$


The trace transformation should be now parameterised as $\tilde{\Lambda}_{b}^{a}=e^{\frac{1}{2} \phi} \delta_{b}^{a}$ to result exactly in Equation (11). Thus, we can conclude that all the three cases can be interpreted as rescalings. The nomenclature we have adopted is justified from the orthonormal perspective ${ }^{4}$.

\section{A Simple Scale-Invariant Theory}

As a warm-up, let us study now a conformally invariant theory by coupling the Ricci scalar $R$ to a scalar field $\psi$, in the metric-affine framework. The nice thing now is that one does not need the existence of an additional gauge field $A_{\mu}$ in order to define the gauge covariant derivative on $\psi$ since torsion and non-metricity offer enough room to accommodate it into them. To be more specific, consider the action

$$
S=\frac{1}{2 \kappa} \int \mathrm{d}^{n} x\left[\sqrt{-g} \psi^{2} R+\lambda \sqrt{-g} g^{\mu \nu} D_{\mu} \psi D_{\nu} \psi\right]
$$

where $\lambda$ is a parameter and $D_{\mu} \psi$ the gauge covariant derivative on the field, to be defined in a moment. Please note that the Dirac-Weyl theory $[60,61]$ corresponds to the above action where the metric-affine $R$ is replaced by the Einstein-Hilbert scalar, the $\lambda$ is fixed to the special value of the conformal coupling, and an additional gauge field $A_{\mu}$ is introduced to define the covariant derivative $D_{\mu}$.

Notice now that the first term in the above action is invariant under conformal transformations of the metric

$$
g_{\mu \nu} \rightarrow \bar{g}_{\mu \nu}=e^{2 \phi} g_{\mu \nu}
$$

provided that we simultaneously transform the scalar field as

$$
\psi \rightarrow \bar{\psi}=e^{\frac{(2-n)}{2} \phi} \psi
$$

To keep this invariance on the kinetic term too, one needs to replace the partial derivative $\partial_{\mu}$ with a covariant one $D_{\mu}=\partial_{\mu}+A_{\mu}$ and also impose a gauge transformation on the field $A_{\mu}\left(A_{\mu} \rightarrow A_{\mu}+\partial_{\mu} \phi\right)$ so as to have the transformation

$$
\bar{D}_{\mu} \bar{\psi}=e^{\frac{(2-n)}{2} \phi} D_{\mu} \psi
$$

and subsequently

$$
\sqrt{-\bar{g}} \bar{g}^{\mu v} \bar{D}_{\mu} \bar{\psi} \bar{D}_{\nu} \bar{\psi}=g^{\mu v} D_{\mu} \psi D_{\nu} \psi
$$

which will ensure the conformal invariance of the total action. Now, what is interesting is that we do not have to add this gauge field $A_{\mu}$ by hand, we have a generalized geometry offering torsion and non-metricity vectors that can do the job. Notice now that since the torsion vector $S_{\mu}$ does not change under conformal transformations, it cannot be regarded as our desired gauge field. The non-metricity (Weyl) vector however, transforms as

$$
\bar{Q}_{\mu}=Q_{\mu}-2 n \partial_{\mu} \phi
$$

under a conformal transformation. Therefore, defining the covariant derivative on the scalar field as

$$
D_{\mu} \equiv \partial_{\mu}+\frac{2-n}{4 n} Q_{\mu}
$$

4 Needless to say, this terminology is not systematically used in the literature, but typically any version that is adopted is referred to as "the conformal transformation". However, our definition of the conformal transformation Equation (12) agrees with the review [7], since the transformation Equation (19) can be seen as the volume changing part of the proper linear transformation Equation (26) (which is appropriately called "the local scale transformation" in [7]) that is generalised "by admitting arbitrary exponents" of the rescaling factor $e^{\phi}$, in the case of Equation (19) in particular giving the metric $\eta_{a b}$ the rescaling weight 2 . In the same way, the frame rescaling Equation (13) is simply the local scale transformation, i.e., the trace part of Equation (26), accompanied with the non-trivial rescaling weight 1 for the frame field and -1 for the coframe field. In the context of torsion transformations, the $\tilde{\delta}$-transformation Equation (13) and the $\bar{\delta}$-transformation Equation (12) correspond to the "strong conformal symmetry" and the "weak conformal symmetry" [14], respectively. 
ensures that Equation (31) is satisfied. So, building the action this way, let us derive the field equations of Equation (27). Variation with respect to the metric tensor yields

$$
-\frac{1}{2} g_{\mu \nu}\left(\psi^{2} R+\lambda(D \psi)^{2}\right)+\psi^{2} R_{(\mu v)}+\lambda D_{\mu} \psi D_{\nu} \psi+\lambda \frac{(n-2)}{2 n} g_{\mu v} \frac{\partial_{\alpha}\left(\sqrt{-g} \psi D^{\alpha} \psi\right)}{\sqrt{-g}}=0
$$

where we have abbreviated $(D \psi)^{2}=g^{\mu v} D_{\mu} \psi D_{\nu} \psi$. Now, since our initial action is conformally invariant one would expect that the trace of the above equation identically vanishes. In fact, the trace of the above equation gives the same equation that one gets when varying with respect to the scalar field $\psi$. Therefore, when the equation of motion for $\psi$ is on shell, the above trace vanishes identically. To see this first note that the trace of the above field equations is

$$
\psi^{2} R+\lambda(D \psi)^{2}-\lambda \frac{\partial_{\alpha}\left(\sqrt{-g} \psi D^{\alpha} \psi\right)}{\sqrt{-g}}=0 .
$$

On the other hand, varying the action with respect to $\psi$, we obtain

$$
R \psi-\lambda \frac{\partial_{\alpha}\left(\sqrt{-g} D^{\alpha} \psi\right)}{\sqrt{-g}}-\lambda \frac{(n-2)}{4 n} Q^{\mu}\left(D_{\mu} \psi\right)=0 .
$$

Multiplying this by $\psi$ (given that $\psi \neq 0$ ) and doing a partial integration it follows that

$$
R \psi^{2}-\lambda \frac{\partial_{\alpha}\left(\sqrt{-g} \psi D^{\alpha} \psi\right)}{\sqrt{-g}}+\lambda\left(\partial_{\mu}+\frac{2-n}{4 n} Q_{\mu}\right) D^{\mu} \psi=0
$$

or equivalently

$$
\psi^{2} R+\lambda(D \psi)^{2}-\lambda \frac{\partial_{\alpha}\left(\sqrt{-g} \psi D^{\alpha} \psi\right)}{\sqrt{-g}}=0
$$

which is indeed the same equation with Equation (35). Lastly, variation of the action with respect to the connection yields

$$
P_{\lambda}^{\mu v}(h)+\lambda \frac{(2-n)}{n} \delta_{\lambda}^{\mu}\left(D^{v} \psi\right)=0
$$

where

$$
P_{\lambda}^{\mu \nu}(h) \equiv-\frac{\nabla_{\lambda}\left(\sqrt{-g} \psi^{2} g^{\mu v}\right)}{\sqrt{-g}}+\frac{\nabla_{\alpha}\left(\sqrt{-g} \psi^{2} g^{\mu \alpha} \delta_{\lambda}^{v}\right)}{\sqrt{-g}}+2 \psi^{2}\left(S_{\lambda} g^{\mu \nu}-S^{\mu} \delta_{\lambda}^{v}-S_{\lambda}^{\mu \nu}\right)
$$

is the Palatini tensor computed with respect to the metric $h_{\mu v}=\psi^{2} g_{\mu v}$. This tensor can also be written as

$$
P_{\lambda}^{\mu \nu}(h)=\psi^{2} P_{\lambda}^{\mu \nu}(g)+\delta_{\lambda}^{v} g^{\mu \alpha} \partial_{\alpha} \psi^{2}-g^{\mu v} \partial_{\lambda} \psi^{2}
$$

where $P_{\lambda}^{\mu v}(g)$ is the usual Palatini tensor computed with respect to the metric tensor $g_{\mu v}$. Looking back at Equation (39), contracting in $\mu=\lambda$ and using the fact that the Palatini tensor is traceless in its first two indices ${ }^{5}$, it follows that

$$
D^{v} \psi=0
$$

which when substituted back at Equation (39) gives

$$
P_{\lambda}{ }^{\mu v}(h)=0 \quad \Rightarrow \quad \psi^{2} P_{\lambda}^{\mu v}(g)=-\delta_{\lambda}^{v} g^{\mu \alpha} \partial_{\alpha} \psi^{2}+g^{\mu v} \partial_{\lambda} \psi^{2}
$$

5 Please note that both $P_{\mu}^{\mu v}(g)=0$ and $P_{\mu}^{\mu \nu}(h)=0$, that is any Palatini tensor that is built from a metric conformally related to $g_{\mu v}$ is also traceless in its first two indices. We shortly return to study this systematically in Appendix B. 
With this at hand we can use the connection decomposition and easily find the affine connection

$$
\Gamma_{\mu \nu}^{\lambda}=\tilde{\Gamma}_{\mu v}+\frac{2}{n-2} g_{\mu \nu} \frac{\partial^{\lambda} \psi}{\psi}-\frac{2}{n-2} \delta_{v}^{\lambda} \frac{\partial_{\mu} \psi}{\psi}+\frac{1}{2} \delta_{\mu}^{\lambda} \tilde{Q}_{v} .
$$

Before finding the expressions for torsion and non-metricity that follow from the above, let us expand Equation (42) to get

$$
\partial_{\mu} \psi-\frac{(n-2)}{4 n} Q_{\mu} \psi=0 \quad \Rightarrow \quad Q_{\mu}=\frac{4 n}{n-2} \frac{\partial_{\mu} \psi}{\psi}
$$

that is, the Weyl vector is exact and powered by the scalar field $\psi$. Now, using the above connection decomposition and the fact that

$$
S_{\mu \nu}{ }^{\lambda}=N^{\lambda}{ }_{[\mu v]} \text { and } Q_{\alpha \mu \nu}=2 N_{(\alpha \mu) v} \text {, where } N^{\lambda}{ }_{\mu \nu} \equiv \Gamma^{\lambda}{ }_{\mu \nu}-\left\{\begin{array}{c}
\lambda \\
\mu v
\end{array}\right\}
$$

it follows that

$$
S_{\mu \nu}{ }^{\lambda}=-2 \frac{\partial_{[\mu} \psi \delta_{v]}^{\lambda}}{\psi}+\frac{1}{2} \delta_{\mu}^{\lambda} \tilde{Q}_{v]} \text { and } Q_{\alpha \mu \nu}=\tilde{Q}_{\alpha} g_{\mu \nu}=\frac{1}{n} Q_{\alpha} g_{\mu \nu}
$$

where the last equality is deduced by the contraction of the previous one. Also recalling that $Q_{\mu}=\frac{4 n}{n-2} \frac{\partial_{\mu} \psi}{\psi}$ we have

$$
Q_{\alpha \mu \nu}=\frac{4}{n-2} g_{\mu \nu} \frac{\partial_{\mu} \psi}{\psi}
$$

which is the case of a Weyl integrable non-metricity. Also, using the above, the torsion tensor may be expressed as

$$
S_{\mu \nu}^{\lambda}=\frac{4}{n-2} \frac{\delta_{[\mu}^{\lambda} \partial_{v]} \psi}{\psi}
$$

with the torsion vector

$$
S_{\mu}=-\frac{2(n-1)}{(n-2)} \frac{\partial_{\mu} \psi}{\psi}
$$

and the above is a case of vectorial torsion with an exact torsion vector. We note at this point that the torsion and the non-metricity are dual to each. This is because they only appear in their vectorial forms in the theory under consideration (and further, pure gauge vectors), which the projective transformations change into each other, and the curvature is projectively invariant. Therefore, from the above construction of the conformally invariant theory, we get the corresponding frame rescaling invariant theory by prescribing the gauge field $A_{\mu}$ defining the covariant derivative of the scalar to be given by torsion instead of non-metricity. To summarise, the three cases of symmetry correspond to the following three prescriptions for the covariant derivative of the scalar field in the action Equation (27).

- projective invariance:

$$
D_{\mu} \equiv \nabla_{\mu} \quad \Rightarrow \quad D_{\mu} \psi=\partial_{\mu} \psi
$$

- conformal invariance:

$$
D_{\mu} \equiv \nabla_{\mu}-\overline{\mathrm{w}}\left(\frac{n-2}{4 n}\right) Q_{\mu} \quad \Rightarrow \quad D_{\mu} \psi=\left[\partial_{\mu}-\left(\frac{n-2}{4 n}\right) Q_{\mu}\right] \psi
$$

- frame rescaling invariance:

$$
D_{\mu} \equiv \nabla_{\mu}-\tilde{\mathrm{w}}\left(\frac{n-2}{n-1}\right) S_{\mu} \Rightarrow D_{\mu} \psi \equiv\left[\partial_{\mu}-\left(\frac{n-2}{n-1}\right) S_{\mu}\right] \psi
$$


In each case, we have written the definition that applies for an arbitrary tensor with the corresponding weight given by the $\mathrm{w}$-symbol, and then specified the action on the rank- 0 tensor $\psi$. For the corresponding projectively invariant theory, the compensating scalar field is not needed at all (if it is included, its transformation should be considered trivial to retain the projective invariance). The duality of torsion and non-metricity vectors in a projectively invariant theory was elaborated in great detail in the recent Reference [23].

Finally, using the above results, the field equations for the scalar field and the metric imply the field equations

$$
R=0 \quad \text { and } \quad R_{\mu v}=0 .
$$

We can now clarify the physical interpretation of the theory Equation (27). It is equivalent to Einstein's Gravity in vacuum, even though the curvature has more degrees of freedom coming from torsion and non-metricity. In this simple conformally invariant model we have a Weyl non-metricity and vectorial torsion both sourced by the scalar field $\psi$, but their role is that of a pure-gauge field. A natural gauge fixing is to choose the transformation parameter $\phi$ such that $\psi=1$ is a constant. This could be seen as the unitary gauge, where both torsion and non-metricity then vanish, and we recover GR with the correct normalisation of the gravitational constant $\kappa$. In any other gauge the gravitational coupling would appear to be a function of time and space, effectively $\kappa \rightarrow \kappa / \psi$, but the physics of the theory would be rendered equivalent by the dynamics of the gauge fields $S_{\mu}$ and $Q_{\mu}$. The equivalence would be broken by adding kinetic terms for these fields, corresponding to the scalars we have listed in the Appendix A. Let us now proceed to the study of those scalars.

\section{The Parity-Even Quadratic Action}

We are interested in quadratic, second order metric-affine theories which are covariant under the three scaling transformations. Let us first note the fact that any curvature scalar ${ }^{6}$ that may be constructed in the Palatini formalism is covariant under conformal transformation and invariant under the pure-gauge projective transformation. Trivially, these curvature scalars are then covariant under the frame transformation as well. Therefore, after the simple example with curvature in Section 3, we shall mostly focus on actions that are quadratic in torsion and non-metricity. Another rationale for our choice of action is that it is the most general quadratic theory involves no derivatives of the connection but is up to second order in derivatives of the metric [71].

\subsection{The Scale-Covariant Scalars}

In this Section we consider the parity-symmetric action in arbitrary spacetime dimension $n$. It is given as

$$
S=\frac{1}{2 \kappa} \int \mathrm{d}^{n} x \sqrt{-g}\left[\mathcal{L}_{Q}^{+}+\mathcal{L}_{T}^{+}+\mathcal{L}_{Q T}^{+}\right]+S_{\text {Matter }}
$$

which is parameterised by the $5+3+3=11$ parameters $a_{i}, b_{i}$ and $c_{i}$ as follows:

$$
\begin{aligned}
\mathcal{L}_{Q}^{+} & =a_{1} Q_{\alpha \mu \nu} Q^{\alpha \mu v}+a_{2} Q_{\alpha \mu \nu} Q^{\mu v \alpha}+a_{3} Q_{\mu} Q^{\mu}+a_{4} q_{\mu} q^{\mu}+a_{5} Q_{\mu} q^{\mu} \\
\mathcal{L}_{T}^{+} & =b_{1} S_{\alpha \mu \nu} S^{\alpha \mu v}+b_{2} S_{\alpha \mu \nu} S^{\mu v \alpha}+b_{3} S_{\mu} S^{\mu} \\
\mathcal{L}_{Q T}^{+} & =c_{1} Q_{\alpha \mu \nu} S^{\alpha \mu \nu}+c_{2} Q_{\mu} S^{\mu}+c_{3} q_{\mu} S^{\mu}
\end{aligned}
$$

For the systematical deduction of this action, we refer the reader to the Appendix A.

To begin with, let us consider how this action can be considered as a limit to GR. We could of course add the Einstein-Hilbert Lagrangian $\mathcal{L}_{E H}=\mathcal{R}-2 \Lambda$ to the above and consider the quadratic

6 There is 1 linear and 10 scalars in the quadratic and parity-even case [65], and in the general case there is an infinite number of such scalars. 
terms as post-Riemannian corrections. Notice now however that for the parameter choice $b_{1}=1$, $b_{2}=-2, b_{3}=-4, a_{i}=0=c_{i}$ one recovers the teleparallel equivalent of GR by imposing a vanishing curvature and non-metricity, as it was shown recently in References [64,72]. It was further shown there that by demanding vanishing curvature and torsion and taking $a_{1}=-a_{3}=1 / 4, a_{2}=-a_{5}=-1 / 2$, $a_{4}=0, b_{i}=0=c_{i}$ one obtains the symmetric teleparallel equivalent of GR $[73,74]$ from the above action. Furthermore if we pick $b_{1}=1, b_{2}=-1, b_{3}=-4, a_{1}=-a_{3}=1 / 4, a_{2}=-a_{5}=-1 / 2$, $a_{4}=0, c_{1}=-c_{2}=c_{3}=2$ and impose only the vanishing of curvature, we may expect to reproduce a generalized equivalent to GR that admits both torsion and non-metricity. The latter possibility has not, however, been considered in detail previously.

Now, in order to obtain a conformally invariant theory we should first restrict the above parameters and find a specific combination for which the total Lagrangian density transforms covariantly under the conformal transformation Equation (12), namely it only picks up a factor $e^{-2 \phi}$. To do so, we use the transformation laws for the quadratic scalars that are derived in detail in the Appendix C.2. Then under a conformal transformation, we have

$$
\begin{aligned}
\overline{\mathcal{L}}_{T}^{+} & =e^{-2 \phi} \mathcal{L}_{T}^{+} \\
\overline{\mathcal{L}}_{Q}^{+} & =e^{-2 \phi} \mathcal{L}_{Q}^{+}-e^{-2 \phi} Q^{\mu} \partial_{\mu} \phi\left(4 a_{1}+4 n a_{3}+2 a_{5}\right)-e^{-2 \phi} q^{\mu} \partial_{\mu} \phi\left(4 a_{2}+4 a_{4}+2 n a_{5}\right) \\
& +e^{-2 \phi}(\partial \phi)^{2} 4\left(n a_{1}+a_{2}+n^{2} a_{3}+a_{4}+n a_{5}\right) \\
\overline{\mathcal{L}}_{\mathrm{Q} T}^{+} & =e^{-2 \phi} \mathcal{L}_{\mathrm{Q} T}^{+}-e^{-2 \phi} 2 S^{\mu} \partial_{\mu} \phi\left(c_{1}+n c_{2}+c_{3}\right) .
\end{aligned}
$$

From these we conclude that the parameter choices fo $a_{i}$ and $c_{i}$ for which

$$
\begin{aligned}
& 0=4 a_{1}+4 n a_{3}+2 a_{5} \\
& 0=4 a_{2}+4 a_{4}+2 n a_{5} \\
& 0=n a_{1}+a_{2}+n^{2} a_{3}+a_{4}+n a_{5} \\
& 0=c_{1}+n c_{2}+c_{3}
\end{aligned}
$$

and whatever $b_{i}^{\prime} s$ guarantee that

$$
\overline{\mathcal{L}}_{\mathrm{Q}}^{+}+\overline{\mathcal{L}}_{T}^{+}+\overline{\mathcal{L}}_{\mathrm{Q} T}^{+}=e^{-2 \phi}\left(\mathcal{L}_{Q}^{+}+\mathcal{L}_{T}^{+}+\mathcal{L}_{Q T}^{+}\right)
$$

as we desired. Only 3 of the above 4 constraints are independent ${ }^{7}$, since $n(56 a)+(56 b)=4(56 c)$. Thus the most general conformally covariant quadratic action is given by $11-3=8$ free parameters.

Now, let us consider frame rescalings Equation (13). Some details of the derivation are found in the Appendix C.3, the result being

$$
\begin{aligned}
\tilde{\mathcal{L}}_{Q}^{+} & =e^{-2 \phi} \mathcal{L}_{Q}^{+} \\
\tilde{\mathcal{L}}_{T}^{+} & =e^{-2 \phi} \mathcal{L}_{T}^{+}-e^{-2 \phi} S^{\mu} \partial_{\mu} \phi\left(2 b_{1}-b_{2}+(n-1) b_{3}\right)+\frac{(n-1)}{4} e^{-2 \phi}(\partial \phi)^{2}\left(2 b_{1}-b_{2}+(n-1) b_{3}\right) \\
\tilde{\mathcal{L}}_{Q T}^{+} & =e^{-2 \phi} \mathcal{L}_{Q T}^{+}-\frac{1}{2} e^{-2 \phi} Q^{\mu} \partial_{\mu} \phi\left(c_{1}+(n-1) c_{2}\right)+\frac{1}{2} e^{-2 \phi} q^{\mu} \partial_{\mu} \phi\left(c_{1}+(1-n) c_{3}\right) .
\end{aligned}
$$

Then, frame rescaling invariance

$$
\tilde{\mathcal{L}}_{Q}^{+}+\tilde{\mathcal{L}}_{T}^{+}+\tilde{\mathcal{L}}_{Q T}^{+}=e^{-2 \phi}\left(\mathcal{L}_{Q}^{+}+\mathcal{L}_{T}^{+}+\mathcal{L}_{Q T}^{+}\right)
$$

7 This is consistent with Equation (A7) in Reference [75]. From their Equation (B4) one sees that the constitutive relation becomes non-invertible in the case of additional conformal symmetry. 
is ensured so long we have $b_{i}{ }^{\prime}$ s and $c_{i}$ 's that satisfy

$$
\begin{aligned}
& 0=2 b_{1}-b_{2}+(n-1) b_{3} \\
& 0=c_{1}+(n-1) c_{2}=0 \\
& 0=c_{1}-(n-1) c_{3}=0
\end{aligned}
$$

and whatever $a_{i}^{\prime}$ s. There are thus 8 independent $\tilde{\delta}$-covariant combinations of scalars.

Now, let us see how our action changes under projective transformations of the connection Equation (11), $\Gamma^{\lambda}{ }_{\mu v} \longrightarrow \hat{\Gamma}^{\lambda}{ }_{\mu v}=\Gamma^{\lambda}{ }_{\mu v}+\delta_{\mu}^{\lambda} \xi_{v}$ which do not affect the spacetime metric $g_{\mu \nu} \longrightarrow \hat{g}_{\mu \nu}=g_{\mu \nu}$. Please note that we do not require the vector $\xi^{\mu}$ to be a gradient. We compute

$$
\begin{aligned}
\hat{\mathcal{L}}_{Q}^{+} & =\mathcal{L}_{Q}^{+}+\left(4 a_{1}+4 n a_{3}+2 a_{5}\right) Q_{\mu} \xi^{\mu}+\left(4 a_{2}+4 a_{4}+2 n a_{5}\right) q_{\mu} \xi^{\mu} \\
& +\left(4 n a_{1}+4 a_{2}+4 n^{2} a_{3}+4 a_{4}+4 n a_{5}\right) \xi_{\mu} \xi^{\mu} \\
\hat{\mathcal{L}}_{T}^{+} & =\mathcal{L}_{T}^{+}-\left[2 b_{1}-b_{2}+(n-1) b_{3}\right] S_{\mu} \xi^{\mu}+\frac{(n-1)}{4}\left[2 b_{1}-b_{2}+(n-1) b_{3}\right] \xi_{\mu} \xi^{\mu} \\
\hat{\mathcal{L}}_{Q T}^{+} & =\mathcal{L}_{Q T}^{+}-\frac{1}{2}\left[c_{1}+(n-1) c_{2}\right] Q_{\mu} \xi^{\mu}+\frac{1}{2}\left[c_{1}-(n-1) c_{3}\right] q_{\mu} \xi^{\mu} \\
& +\left(c_{1}+n c_{2}+c_{3}\right)\left[2 S_{\mu} \xi^{\mu}-(n-1) \xi_{\mu} \xi^{\mu}\right] .
\end{aligned}
$$

Therefore, the total action changes according to

$$
\begin{aligned}
\hat{\mathcal{L}}_{Q}^{+}+\hat{\mathcal{L}}_{T}^{+}+\hat{\mathcal{L}}_{\mathrm{Q} T}^{+} & =\mathcal{L}_{Q}^{+}+\mathcal{L}_{T}^{+}+\mathcal{L}_{Q T}^{+} \\
& +\left[2\left(2 a_{1}+2 n a_{3}+a_{5}\right)-\frac{1}{2}\left(c_{1}+(n-1) c_{2}\right)\right] Q_{\mu} \xi^{\mu} \\
& +\left[2\left(2 a_{2}+2 a_{4}+n a_{5}\right)+\frac{1}{2}\left(c_{1}-(n-1) c_{3}\right)\right] q_{\mu} \xi^{\mu} \\
& +\left[-2 b_{1}+b_{2}-(n-1) b_{3}+2\left(c_{1}+n c_{2}+c_{3}\right)\right] S_{\mu} \xi^{\mu} \\
& +\left[4\left(n a_{1}+a_{2}+n^{2} a_{3}+a_{4}+n a_{5}\right)+\frac{(n-1)}{4}\left(2 b_{1}-b_{2}+(n-1) b_{3}-4\left(c_{1}+n c_{2}+c_{3}\right)\right)\right] \xi_{\mu} \xi^{\mu}
\end{aligned}
$$

Then, projective invariance is ensured if the parameters satisfy

$$
\begin{aligned}
& 0=4\left(2 a_{1}+2 n a_{3}+a_{5}\right)-c_{1}-(n-1) c_{2} \\
& 0=4\left(2 a_{2}+2 a_{4}+n a_{5}\right)+c_{1}-(n-1) c_{3} \\
& 0=2 b_{1}-b_{2}+(n-1) b_{3}-2\left(c_{1}+n c_{2}+c_{3}\right) \\
& 0=16\left(n a_{1}+a_{2}+n^{2} a_{3}+a_{4}+n a_{5}\right)+(n-1)\left(2 b_{1}-b_{2}+(n-1) b_{3}-4\left(c_{1}+n c_{2}+c_{3}\right)\right)
\end{aligned}
$$

Only 3 out of the first 4 constraints are independent. The important thing to note here is that the parameters $a_{i}, b_{i}, c_{i}$ mix when one demands projective invariance. This means that $\mathcal{L}_{Q^{\prime}}^{+} \mathcal{L}_{T}^{+}$and $\mathcal{L}_{\mathrm{Q} T}^{+}$may not independently projective invariant though their sum is. This was not the case when we considered conformal and frame rescaling transformations where the parameters did not mix and $\mathcal{L}_{\mathrm{Q}}^{+}, \mathcal{L}_{T}^{+}$and $\mathcal{L}_{\mathrm{Q} T}^{+}$were all independently invariant under the associated transformations iff their sum was. 


\subsection{The Field Equations}

Having restricted the parameter space in the each of the three cases we can now obtain an invariant theory by coupling the above to $\psi^{2}$. We first combine the case of conformal and frame rescaling transformations in a single action given by

$$
S=\frac{1}{2 \kappa} \int \mathrm{d}^{n} x \sqrt{-g}\left[\psi^{2}\left(\mathcal{L}_{\mathrm{Q}}^{+}+\mathcal{L}_{\mathrm{Q} T}^{+}+\mathcal{L}_{T}^{+}\right)+\lambda g^{\mu v} D_{\mu} \psi D_{v} \psi\right]
$$

where again $\lambda$ is a parameter, $D_{\mu}$ is the gauge covariant derivative to be defined later, and $\mathcal{L}_{Q}^{+}+\mathcal{L}_{T}^{+}+\mathcal{L}_{\mathrm{Q} T}^{+}$was specified in Equation (54). Now, it will be convenient for the calculations to define the "superpotentials"

$$
\begin{aligned}
\Omega^{\alpha \mu v} & \equiv a_{1} Q^{\alpha \mu v}+a_{2} Q^{\mu v \alpha}+a_{3} g^{\mu v} Q^{\alpha}+a_{4} g^{\alpha \mu} q^{v}+a_{5} g^{\alpha \mu} Q^{v} \\
\Sigma^{\alpha \mu v} & \equiv b_{1} S^{\alpha \mu v}+b_{2} S^{\mu v \alpha}+b_{3} g^{\mu v} S^{\alpha} \\
\Pi^{\alpha \mu v} & \equiv c_{1} S^{\alpha \mu v}+c_{2} g^{\mu v} S^{\alpha}+c_{3} g^{\alpha \mu} S^{v}
\end{aligned}
$$

for non-metricity, torsion and their mixing, respectively. With these, the above are written as

$$
\begin{aligned}
\mathcal{L}_{Q}^{+} & =Q_{\alpha \mu \nu} \Omega^{\alpha \mu v} \\
\mathcal{L}_{T}^{+} & =S_{\alpha \mu \nu} \Sigma^{\alpha \mu \nu} \\
\mathcal{L}_{Q T}^{+} & =Q_{\alpha \mu v} \Pi^{\alpha \mu \nu} .
\end{aligned}
$$

We are now in a position to derive the variations of the above. Some intermediate steps in the derivations are confined to the Appendix B. Let us first compute variations with respect to the metric. We have

$$
\sqrt{-g} \psi^{2} \delta_{g} \mathcal{L}_{Q}^{+}=\left(\delta g^{\mu \nu}\right)\left[\sqrt{-g} \psi^{2} L_{(\mu v)}+\left(2 S_{\lambda}-\nabla_{\lambda}\right) J_{(\mu \nu)}^{\lambda}+g_{\mu v}\left(2 S_{\lambda}-\nabla_{\lambda}\right) \zeta^{\lambda}+\alpha_{4}\left(2 S_{(\mu}-\nabla_{(\mu}\right)\left(\sqrt{-g} \psi^{2} q_{\nu)}\right)\right]
$$

where

$$
\begin{aligned}
L_{\mu v} & \equiv\left(a_{1} Q_{\mu \alpha \beta}+a_{2} Q_{\alpha \beta \mu}\right) Q_{v}{ }^{\alpha \beta}+\left(a_{3} Q_{\mu}+a_{5} q_{\mu}\right) Q_{v}+a_{3} Q_{\alpha \mu \nu} Q^{\alpha} \\
& +Q_{\mu v \alpha}\left(a_{4} q^{\alpha}+a_{5} Q^{\alpha}\right)-\Omega^{\alpha \beta}{ }_{v} Q_{\alpha \beta \mu}-\Omega_{\alpha \mu \beta} Q^{\alpha \beta}{ }_{v}
\end{aligned}
$$

and we have also defined the tensor densities

$$
\begin{gathered}
J_{(\mu v)}^{\lambda} \equiv \sqrt{-g} \psi^{2}\left(\alpha_{1} Q_{\mu \nu}^{\lambda}+a_{2} Q_{\mu \nu}{ }^{\lambda}+\Omega_{\mu \nu}^{\lambda}\right) \\
\zeta^{\lambda} \equiv \sqrt{-g} \psi^{2}\left(a_{3} Q^{\lambda}+a_{5} q^{\lambda}\right) .
\end{gathered}
$$

Continuing with the pure torsion and mixed part, we obtain

$$
\sqrt{-g} \psi^{2} \delta_{g} \mathcal{L}_{T}^{+}=\left(\delta g^{\mu \nu}\right) \sqrt{-g} \psi^{2}\left[b_{1}\left(2 S_{v \alpha \beta} S_{\mu}^{\alpha \beta}-S_{\alpha \beta \mu} S^{\alpha \beta}{ }_{v}\right)-b_{2} S_{v \alpha \beta} S_{\mu}^{\alpha \beta}+b_{3} S_{\mu} S_{v}\right]
$$

and

$$
\begin{aligned}
\sqrt{-g} \psi^{2} \delta_{g} \mathcal{L}_{Q T}^{+} & =\left(\delta g^{\mu \nu}\right) \sqrt{-g} \psi^{2}\left[\Pi_{\mu \alpha \beta} Q_{v}{ }^{\alpha \beta}\right. \\
& \left.-\left(c_{1} S_{\alpha \beta \nu} Q^{\alpha \beta}{ }_{\mu}+c_{2} S^{\alpha} Q_{\alpha \mu \nu}+c_{3} S^{\alpha} Q_{\mu \nu \alpha}\right)+\frac{1}{\sqrt{-g} \psi^{2}}\left(2 S_{\lambda}-\nabla_{\lambda}\right)\left(\sqrt{-g} \psi^{2} \Pi^{\lambda}{ }_{\mu \nu}\right)\right]
\end{aligned}
$$

Using all the above we can now derive the field equations for the conformally and frame rescaling invariant theories. To obtain a conformally invariant theory, the parameters must satisfy Equation (56) and the gauge covariant derivative on the scalar field has to be defined a in Equation (51b). 
On the other hand, in order to obtain a frame rescaling invariant theory, the parameter space is restricted by the constraints Equation (60) and the gauge covariant derivative should be defined as in Equation (51c).

Having clarified this, the field equations after varying with respect to the metric tensor are

$$
\psi^{2}\left(Z_{(\mu \nu)}-\frac{1}{2} g_{\mu v} \mathcal{L}\right)-\frac{1}{2} g_{\mu \nu} \lambda(D \psi)^{2}+\lambda\left(D_{\mu} \psi D_{\nu} \psi+K_{\mu \nu}\right)=0
$$

where

$$
\begin{aligned}
Z_{\mu v} & \equiv L_{\mu v}+\xi_{\mu v}+b_{1}\left(2 S_{v \alpha \beta} S_{\mu}^{\alpha \beta}-S_{\alpha \beta \mu} S^{\alpha \beta}{ }_{v}\right)-b_{2} S_{v \alpha \beta} S_{\mu}{ }^{\alpha \beta}+b_{3} S_{\mu} S_{v}+\Pi_{\mu \alpha \beta} Q_{v}{ }^{\alpha \beta} \\
& -\left(c_{1} S_{\alpha \beta \nu} Q^{\alpha \beta}{ }_{\mu}+c_{2} S^{\alpha} Q_{\alpha \mu \nu}+c_{3} S^{\alpha} Q_{\mu \nu \alpha}\right)+\frac{1}{\sqrt{-g} \psi^{2}}\left(2 S_{\lambda}-\nabla_{\lambda}\right)\left(\sqrt{-g} \psi^{2} \Pi^{\lambda}{ }_{\mu \nu}\right)
\end{aligned}
$$

and

$$
\xi_{\mu \nu} \equiv \frac{1}{\sqrt{-g} \psi^{2}}\left[\left(2 S_{\lambda}-\nabla_{\lambda}\right) J_{(\mu v)}^{\lambda}+g_{\mu \nu}\left(2 S_{\lambda}-\nabla_{\lambda}\right) \zeta^{\lambda}+\alpha_{4}\left(2 S_{(\mu}-\nabla_{(\mu}\right)\left(\sqrt{-g} \psi^{2} q_{\nu)}\right)\right] .
$$

Since

$$
\int \mathrm{d}^{n} x \sqrt{-g} K_{\mu \nu} \equiv \int \mathrm{d}^{n} x \sqrt{-g}\left(D^{\alpha} \psi\right) \frac{\delta\left(D_{\alpha} \psi\right)}{\delta g^{\mu \nu}}
$$

we have therefore

$$
K_{\mu \nu}=\frac{(n-2)}{2 n} g_{\mu \nu} \frac{\partial_{\alpha}\left(\sqrt{-g} \psi D^{\alpha} \psi\right)}{\sqrt{-g}}
$$

for the conformally invariant theory and

$$
K_{\mu v}=0
$$

for the frame rescaling invariant theory ${ }^{8}$.

Let us continue with the rest of the field equations. Variation with respect to the connection gives

$$
\psi^{2}\left({H^{\mu v}}_{\lambda}+\delta_{\lambda}^{\mu} k^{v}+\delta_{\lambda}^{v} h^{\mu}+g^{\mu v} h_{\lambda}+f^{[\mu} \delta_{\lambda}^{v]}\right)+\Theta_{\lambda}^{\mu v}=0
$$

where

$$
\begin{aligned}
H_{\lambda}^{\mu v} & \equiv a_{1} Q_{\lambda}^{\nu \mu}+2 a_{2}\left(Q^{\mu v}{ }_{\lambda}+Q_{\lambda}{ }^{\mu v}\right)+2 b_{1} S_{\lambda}^{\mu \nu}+2 b_{2} S_{\lambda}{ }^{[\mu v]}+c_{1}\left(S^{\nu \mu}{ }_{\lambda}-S_{\lambda}{ }^{v \mu}+Q^{[\mu v]} \lambda\right. \\
k_{\mu} & \equiv 4 a_{3} Q_{\mu}+2 a_{5} q_{\mu}+2 c_{2} S_{\mu} \\
h_{\mu} & \equiv a_{5} Q_{\mu}+2 a_{4} q_{\mu}+c_{3} S_{\mu} \\
f_{\mu} & \equiv c_{2} Q_{\mu}+c_{3} q_{\mu}+2 b_{3} S_{\mu}
\end{aligned}
$$

and

$$
\Theta_{\lambda}^{\mu v} \equiv \frac{\partial}{\partial \Gamma^{\lambda}{ }_{\mu \nu}}\left(\lambda g^{\alpha \beta} D_{\alpha} \psi D_{\beta} \psi\right)
$$

which for the conformally invariant case takes the form

$$
\Theta_{\lambda}^{\mu v}=-\lambda\left(\frac{n-2}{n}\right) \psi\left(D^{v} \psi\right) \delta_{\lambda}^{\mu}
$$

and for the frame rescaling invariant theory

8 This is so because in this case the gauge covariant derivative is constructed in terms of $S_{\mu}$ and the latter is independent of the metric tensor. 


$$
\Theta_{\lambda}^{\mu \nu}=-2 \lambda\left(\frac{n-2}{n-1}\right) \psi\left(D^{[\mu} \psi\right) \delta_{\lambda}^{v]}
$$

since the gauge covariant derivative given by Equation (51b) for the former and Equation (51c) for the latter respectively. To conclude, if we neglect the coupling via the covariant derivative, we obtain

$$
\psi^{2}\left(H_{\lambda}^{\mu v}+\delta_{\lambda}^{\mu} k^{v}+\delta_{\lambda}^{v} h^{\mu}+g^{\mu v} h_{\lambda}+f^{[\mu} \delta_{\lambda}^{v]}\right)=0
$$

however, for the conformally invariant case the $\Gamma$-field equations read

$$
\psi^{2}\left(H_{\lambda}^{\mu v}+\delta_{\lambda}^{\mu} k^{\nu}+\delta_{\lambda}^{v} h^{\mu}+g^{\mu v} h_{\lambda}+f^{[\mu} \delta_{\lambda}^{v]}\right)=\lambda\left(\frac{n-2}{n}\right) \psi\left(D^{v} \psi\right) \delta_{\lambda}^{\mu}
$$

and for the frame rescaling invariant case

$$
\psi^{2}\left(H_{\lambda}^{\mu \nu}+\delta_{\lambda}^{\mu} k^{\nu}+\delta_{\lambda}^{v} h^{\mu}+g^{\mu v} h_{\lambda}+f^{[\mu} \delta_{\lambda}^{v]}\right)=2 \lambda\left(\frac{n-2}{n-1}\right) \psi\left(D^{[\mu} \psi\right) \delta_{\lambda}^{v]}
$$

Now, to close the system of the field equations it remains to vary with respect to the scalar $\psi$. Neglecting the coupling via the covariant derivative, we would obtain simply

$$
\psi \mathcal{L}=\lambda \square \phi
$$

but in the conformally invariant case we find

$$
\psi \mathcal{L}=\lambda\left(\frac{n-2}{4 n} Q_{\mu} D^{\mu} \psi+\frac{\partial_{\mu}\left(\sqrt{-g} D^{\mu} \psi\right)}{\sqrt{-g}}\right)
$$

while for the frame rescaling invariant theory, one obtains

$$
\psi \mathcal{L}=\lambda\left(\frac{n-2}{n-1} S_{\mu} D^{\mu} \psi+\frac{\partial_{\mu}\left(\sqrt{-g} D^{\mu} \psi\right)}{\sqrt{-g}}\right) .
$$

\begin{tabular}{|c|c|c|c|c|c|c|}
\hline Invariance & Constraints & $D_{\mu}=\partial_{\mu}-A_{\mu}$ & $g$-EoM & Г-EoM & $\psi$-EoM & Total \\
\hline projective & Equation (63) & & Equation (73) where $K_{\mu v}=0$ & Equation (82a) & Equation (83a) & Equation (96) \\
\hline conformal & Equation (56) & $A_{\mu}=\left(\frac{n-2}{4 n}\right) Q_{\mu}$ & Equation (73) with Equation (77a) & Equation (82b) & Equation (83b) & Equation (92) \\
\hline
\end{tabular}

We have summarised the results in Table 2 below.

Table 2. The invariant actions (given by Equation (53) by taking into account the constraints and the prescription for the derivative) and the equations of motion. The final column refers to the parameter constraints on the total action Equation (87) to be derived in Section 5.

Let us examine Equations (82b) and (82c) a little further. To do so, notice that we can consider three operations on Equations (82b) and (82c). We can contract in $\mu=\lambda$, contact in $\nu=\lambda$ and multiply (and contact) by $g^{\mu \nu}$. Then we get three vector equations that we may formally write as

$$
\begin{aligned}
\alpha_{1} Q_{\mu}+\alpha_{2} q_{\mu}+\alpha_{3} S_{\mu} & =\frac{\partial_{\mu} \psi}{\psi} \\
\beta_{1} Q_{\mu}+\beta_{2} q_{\mu}+\beta_{3} S_{\mu} & =\frac{\partial_{\mu} \psi}{\psi} \\
\gamma_{1} Q_{\mu}+\gamma_{2} q_{\mu}+\gamma_{3} S_{\mu} & =\frac{\partial_{\mu} \psi}{\psi}
\end{aligned}
$$


where the $\alpha_{i}, \beta_{i}, \gamma_{i}$ are all combinations of $a_{i}, b_{i}, c_{i}$ and $\lambda$. Then the above system of equations can be formally solved ${ }^{9}$ to give

$$
Q_{\mu}=\lambda_{1} \frac{\partial_{\mu} \psi}{\psi} q_{\mu}=\lambda_{2} \frac{\partial_{\mu} \psi}{\psi} S_{\mu}=\lambda_{3} \frac{\partial_{\mu} \psi}{\psi}
$$

where the $\lambda_{i}^{\prime} s$ depend on $\alpha_{i}, \beta_{i}, \gamma_{i}$. This result, when substituted back at Equations (82b) and (82c), yields

$$
H_{\lambda}^{\mu v}=\sigma_{1} \delta_{\lambda}^{\mu} \frac{\partial_{\nu} \psi}{\psi}+\sigma_{2} \delta_{\lambda}^{\nu} \frac{\partial_{\mu} \psi}{\psi}+\sigma_{3} g^{\mu \nu} \frac{\partial^{\lambda} \psi}{\psi}
$$

where again the $\sigma_{i}^{\prime} s$ depend on $a_{i}, b_{i}, c_{i}$ and $\lambda$. These manipulations make it clear that just as in the simple case studied in Section 3, the non-metricity and torsion remain pure gauge in the case of the generic quadratic action which does not include the derivatives of the connection.

\section{The General Quadratic Theory}

In this Section we shall first complete the analysis of the previous Section by incorporating the parity-odd invariants. Then, in Section 5.2, we comment on possible applications of our new results, in particular we point out the various different frameworks wherein it could be interesting to build scale-invariant theories: considering post-Riemannian corrections to (a scale-free version of) the Einstein-Hilbert action, giving kinetic terms to the connection, or constructing scale-invariant teleparallel theories.

\subsection{The Parity-Odd Scalars}

We now specialise to $n=4$ and take into account also the possible CP-violating terms, which were derived in the Appendix A. We may thus write the action as

$$
S=\frac{1}{2 \kappa} \int \mathrm{d}^{4} x \sqrt{-g}\left[\psi^{2}\left(\mathcal{L}_{Q}+\mathcal{L}_{\mathrm{Q} T}+\mathcal{L}_{T}\right)+\lambda g^{\mu \nu} D_{\mu} \psi D_{\nu} \psi+\psi^{4} \Lambda\right]
$$

where, for generality one could consider the quartic self-interaction (that in the $\psi=1$ gauge is seen just as the cosmological constant, and can be neglected in the following as irrelevant to our discussion), and the three pieces of Lagrangians are now understood as $\mathcal{L}_{Q}=\mathcal{L}_{Q}^{+}+\mathcal{L}_{Q}^{-}, \mathcal{L}_{T}=\mathcal{L}_{T}^{+}+\mathcal{L}_{T}^{-}$, and $\mathcal{L}_{\mathrm{Q} T}=\mathcal{L}_{\mathrm{Q} T}^{+}+\mathcal{L}_{\mathrm{Q} T}^{-}$, where the parity-even contributions had been specified in Equation (54), and the parity-odd contributions are given as follows:

$$
\begin{aligned}
\mathcal{L}_{Q}^{-} & \equiv a_{6} \epsilon^{\alpha \beta \gamma \delta} Q_{\alpha \beta \mu} Q_{\gamma \delta}{ }^{\mu}=a_{6} A_{6} \\
\mathcal{L}_{T}^{-} & \equiv b_{5} S_{\mu} t^{\mu}+b_{6} \epsilon^{\alpha \beta \gamma \delta} S_{\alpha \beta \mu} S_{\gamma \delta}{ }^{\mu}=b_{5} B_{5}+b_{6} B_{6} \\
\mathcal{L}_{Q T}^{-} & \equiv c_{4} Q_{\mu} t^{\mu}+c_{5} q^{\mu} t_{\mu}+c_{6} \epsilon^{\alpha \beta \gamma \delta} Q_{\alpha \beta \mu} S_{\gamma \delta}{ }^{m u}=c_{4} C_{4}+c_{5} C_{5}+c_{6} C_{6}
\end{aligned}
$$

Let us now find the parameter space for the above action to be invariant under each of the three transformations, starting with the conformal transformation Equation (12). The newly added parity-odd terms transform as

$$
\bar{A}_{6}=e^{-2 \phi} A_{6}, \quad \bar{B}_{5}=e^{-2 \phi} B_{5}, \quad \bar{B}_{6}=e^{-2 \phi} B_{6}
$$

and

$$
\bar{C}_{4}=e^{-2 \phi}\left(C_{4}-8 t^{\mu} \partial_{\mu} \phi\right), \quad \bar{C}_{5}=e^{-2 \phi}\left(C_{5}-2 t^{\mu} \partial_{\mu} \phi\right), \quad \bar{C}_{6}=e^{-2 \phi}\left(C_{6}-2 t^{\mu} \partial_{\mu} \phi\right)
$$

9 Assuming that the determinant of the matrix corresponding to the system does not vanish. 
under a conformal metric transformation. Thus, the only mixed parity-odd terms transform non-covariantly. We may write simply that

$$
\begin{aligned}
\overline{\mathcal{L}}_{Q}^{-} & =e^{-2 \phi} \mathcal{L}_{Q}^{-} \\
\overline{\mathcal{L}}_{T}^{-} & =e^{-2 \phi} \mathcal{L}_{T}^{-} \\
\overline{\mathcal{L}}_{Q T}^{-} & =e^{-2 \phi} \mathcal{L}_{Q}^{-}-2 e^{-2 \phi} t^{\mu}\left(\partial_{\mu} \phi\right)\left(4 c_{4}+c_{5}+c_{6}\right) .
\end{aligned}
$$

The transformation for the parity-even part of the Lagrangian we have already computed in the previous section. So, for the total action to be invariant under conformal transformations in $n=4$ we must have

$$
\begin{aligned}
& 0=2 a_{1}+8 a_{3}+a_{5} \\
& 0=a_{2}+a_{4}+2 a_{5} \\
& 0=4 a_{1}+a_{2}+16 a_{3}+a_{4}+4 a_{5} \\
& 0=c_{1}+4 c_{2}+c_{3} \\
& 0=4 c_{4}+c_{5}+c_{6} .
\end{aligned}
$$

Please note that the first four constraints (of which only three are independent) in the above are the ones we had derived previously for the pure parity-even Lagrangian and the last constraint is imposed on the parity-odd part. We should mention that the additional constraint establishes a relation only between the coefficients of the parity-odd terms and does not mix them with the parameters of the parity-even scalars.

Now, under a frame rescaling Equation (13) the parity-odd parts transform as

$$
\begin{aligned}
\tilde{\mathcal{L}}_{Q}^{-} & =e^{-2 \phi} \mathcal{L}_{Q}^{-} \\
\tilde{\mathcal{L}}_{T}^{-} & =e^{-2 \phi} \mathcal{L}_{T}^{-}-\frac{1}{2} e^{-2 \phi} t^{\mu} \partial_{\mu} \phi\left(3 b_{5}+4 b_{6}\right) \\
\tilde{\mathcal{L}}_{\mathrm{Q} T}^{-} & =e^{-2 \phi} \mathcal{L}_{Q}^{-} .
\end{aligned}
$$

And for the total action to be invariant under the frame rescalings, the parameters must satisfy

$$
\begin{aligned}
& 0=2 b_{1}-b_{2}+3 b_{3}=0 \\
& 0=c_{1}+3 c_{2} \\
& 0=c_{1}-3 c_{3} \\
& 0=3 b_{5}+4 b_{6} .
\end{aligned}
$$

Again, the first three constraints above are the same with the pure parity-even theory and the last one is imposed among the parameters of the parity-odd terms.

Now, in order to study the parameter space for the projective invariant case, again there is no need for a scalar field $\psi$ to compensate for the invariance (and if it is included, it should not be considered charged under the transformation). By Equation (11), the parity-odd parts transform according to

$$
\begin{aligned}
\hat{\mathcal{L}}_{Q}^{-} & =\mathcal{L}_{Q}^{-} \\
\hat{\mathcal{L}}_{T}^{-} & =\mathcal{L}_{T}^{-}-\frac{3}{2} b_{5} t_{\mu} \xi^{\mu}-2 b_{6} t_{\mu} \xi^{\mu} \\
\hat{\mathcal{L}}_{\mathrm{Q} T}^{-} & =\mathcal{L}_{\mathrm{Q} T}^{-}+2 t_{\mu} \xi^{\mu}\left(4 c_{4}+c_{5}+c_{6}\right)
\end{aligned}
$$

as can be easily checked. So, projective invariance of the total action is ensured if the parameters satisfy 


$$
\begin{aligned}
& 0=4\left(2 a_{1}+8 a_{3}+a_{5}\right)-c_{1}-3 c_{2} \\
& 0=4\left(2 a_{2}+2 a_{4}+4 a_{5}\right)+c_{1}-3 c_{3} \\
& 0=-2 b_{1}+b_{2}-3 b_{3}+2\left(c_{1}+4 c_{2}+c_{3}\right) \\
& 0=16\left(4 a_{1}+a_{2}+16 a_{3}+a_{4}+4 a_{5}\right)-3\left(2 b_{1}-b_{2}+3 b_{3}-4\left(c_{1}+4 c_{2}+c_{3}\right)\right) \\
& 0=-3 b_{5}-4 b_{6}+16 c_{4}+4 c_{5}+4 c_{6} .
\end{aligned}
$$

Please note that in comparison with the pure parity-even case, the first four constraints remain the same (and they are not linearly independent), and a fifth additional constraint is imposed only among the parameters of the parity-odd scalars. The important thing is that the constraints again do not mix the parameters of the parity-even with the parameters of the parity-odd scalars.

Having completed the derivation of the scale-invariant theories, let us have a brief look at their generic properties. Let $T_{\mu \nu}$ be the stress-energy tensor and $H^{\alpha}{ }_{\mu \nu}$ the hypermomentum tensor for the matter fields. The two sets of field equations in any Palatini theory can then be written as

$$
M_{\mu v}=T_{\mu v}
$$

and

$$
\Xi^{\alpha}{ }_{\mu \nu}=H^{\alpha}{ }_{\mu v} .
$$

As it is shown in detail in the Appendix B, the three versions of scale symmetry imply certain properties for these tensors. The conformal symmetry is associated with tracelessness. That is, for the theory to be conformally invariant, a necessary requirement is that $M \equiv M^{\alpha}{ }_{\alpha}=T^{\alpha}{ }_{\alpha} \equiv T=0$. A basic property of the Maxwell field and the massless fermion is that their energy-momentum tensors are traceless. Of course, the Proca field and the massive fermion break scale invariance by introducing the mass scale. Now, it is interesting to note that the projective symmetry on the other hand implies the tracelessness of the hypermomentum, in particular that $\Xi^{\mu} \equiv \Xi_{\alpha}{ }^{\alpha \mu}=H_{\alpha}{ }^{\alpha \mu} \equiv H^{\mu}=0$. The fermion is projectively invariant, but the Maxwell (or Proca) field is invariant only under the symmetric teleparallel projection (when we assume the minimal coupling principle $\partial_{\mu} \rightarrow \nabla_{\mu}$ of the Palatini formalism). Finally, the invariance of the theory under the frame rescalings implies the identities

$$
M=-\frac{\partial_{\mu}\left(\sqrt{-g} \Xi^{\mu}\right)}{2 \sqrt{-g}} \Rightarrow T=-\frac{\partial_{\mu}\left(\sqrt{-g} H^{\mu}\right)}{2 \sqrt{-g}} .
$$

Interestingly, this version of scale symmetry can be compatible with a matter source that has a trace, given that the matter source also possesses hypermomentum.

\subsection{On Applications to Theory}

As discussed in the introduction Section 1, there is a vast amount of possible applications for scale-invariant theories. In fact, some may contemplate whether this symmetry should be finally promoted to the same foundational status as the Lorentz symmetry, or perhaps even more properly the general coordinate invariance, to which it is in some sense related as the multiplication to the addition. In any case, in this paper, we have focused on formal developments, and will return to specific applications elsewhere.

However, it is pertinent to clarify in some detail how our results might be useful in various contexts of gravitational theory model building. At face value, our quadratic actions have trivial predictions. Since we have not added kinetic terms to the connections, the quadratic action $\mathcal{L}_{Q}+\mathcal{L}_{T}+\mathcal{L}_{Q T}$ for any choice of parameters is but a generalised mass term, something like $\sim \Gamma^{2}$. This does not give the connection any dynamics, but the solutions for the $\Gamma$ are pure-gauge, as was the case for the distortion in the explicitly solved simple example of Section 3. Indeed we had sketched how one arrives at the same conclusion in the end of Section 4 . Now, one can of course add the linear curvature action of 
Section 3 to the quadratic action, and then the connection does get dynamics, those of $\mathrm{GR}^{10}$. The reason is that though the curvature includes derivatives of the connection, they become mere boundary terms in the case of the action that is linear in the curvature. However, non-trivial dynamics could be achieved by taking into account quadratic invariants of curvature (which, we recall, are all invariant in $n=4$ under the three types of scale gauge transformations). In the context of considering corrections to the scale-invariant version of the Einstein-Cartan theory, the "Einstein-Cartan-Weyl-Dirac" action Equation (27), it seems to be a perfectly natural to include quadratic curvature terms besides the quadratic contribution $\psi^{2}\left(\mathcal{L}_{Q}+\mathcal{L}_{T}+\mathcal{L}_{Q T}\right)$.

On the other hand, we are now fully armed with the scale-invariant arsenal to set the torsion and the non-metricity propagating directly by including their derivatives into the action. In the previous subsection we had completed the derivation of the covariant scalars, and the scale-covariant derivatives acting on scalars we had already deduced in Section 3. From these ingredients, we can straighforwardly construct non-integrable scale-invariant geometries. In such a case, we would incorporate kinetic terms for the gauge field $A_{\mu}$ (which, recall, is the non-metricity trace a.k.a. Weyl vector in the case of conformal and the torsion trace in the case of frame rescaling invariance).

However, it can be even more interesting to constrain the dynamics of the connection, instead of (or perhaps, in addition to) adding kinetic terms via curvature terms or covariant derivatives. In teleparallism, we supplement the action using the Lagrange multiplier $\lambda_{\alpha} \beta \mu v$ that transforms covariantly under both the general coordinate and the scale transformation. The term $\lambda_{\alpha}{ }^{\beta \mu v} R^{\alpha} \beta \mu v$ then does not break the invariance, but appropriately restricts the rotational part of connection to be pure gauge, i.e., forces the $\Gamma$ to be flat. In the metric-compatible case, we should add a further Lagrange multiplier that sets the non-metricity tensor to vanish, and it turns out that, quite interestingly, even without adding the explicit derivatives, the torsion obtains its dynamics via such a mechanism ${ }^{11}[64,72]$. It was known that there is a unique parameter combination that yields the teleparallel equivalent of GR, and from the results of this paper we see that there is a 3-parameter class of models that is invariant under frame rescalings, and a full 5-parameter class of models that is conformally invariant (in both cases, we are not counting the overall normalisation of the action but are taking into account the parameter $\lambda$ ). Please note that the frame rescaling has to be understood now in its teleparallel version reported in Table 1 i.e., only the antisymmetric part of the connection enjoys the projection, if one wants to confine to the strictly metric-compatible context.

On the other hand, one may augment the flatness constraint with the constraint of vanishing torsion, leading then to symmetric teleparallelism, see [75,77-82] for current studies into such geometry. This is a totally different perspective to the theory of gravity, wherein the spacetime affine connection can be actually fully eliminated, as was only recently clarified in [64,72]. We may thus in principle "purify" gravitation from (non-integrable) spacetime geometry, and the possibly profound implications of this insight certainly call for further investigation. It was known that the parameter combination of the non-metricity scalars that leads to the equivalent of GR in such a geometry is unique, and that it is exclusively for this combination that the affine connection, to the linear order, in addition to being pure gauge, decouples from the action [72]. From the results of this paper we may add that this combination, as in fact any other, is also covariant under the frame rescaling wherein only the

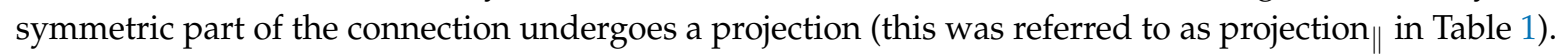
However, under the conformal transformation Equation (12) there exists only a four-parameter class of covariant scalars which is second order in the derivatives of the metric. Promoting the covariant scalars

10 If there is hypermomentum, the situation changes, though the connection remains undynamical, as indeed is known from the seminal example of the Einstein-Cartan-Sciama-Kibble theory. Adding non-minimal derivative interactions of the connection to the matter sector could of course make the connection dynamical.

11 An alternative method is the Golovnev's "inertial variation" [76] wherein instead of the connection one varies a gauge transformation parameter. However, it is against the spirit of the Palatini formalism to set the connection a priori into the purely inertial form. 
into invariant ones with the aid of the dilaton and including the associated parameter $\lambda$, one can easily verify that the symmetric teleparallel equivalent of GR is included among the conformally invariant quadratic theories.

\section{Conclusions}

After a century since its introduction, we considered it timely to revisit the formulation of a scale-invariant theory of gravity, in particular in view of the consistent and viable theories that may be constructed from invariants of non-metricity, torsion, and both.

Scale transformations in metric-affine geometry have been considered previously. For example, in Section 6.1 of the review [7], in addition to the curvature sector which is more trivial, the basic properties of the quadratic non-metric scalars and torsion scalars are clarified, i.e., that the torsion trace squared has a different scale-covariance property than the tensorial and the axial invariant, and the volume-changing non-metricity invariant has a different scale-covariance property than the volume-preserving invariants. The points of departure in this paper were that $(0)$ we presented the analysis in the Palatini formalism. Though the exterior calculus is elegant and makes some aspects of the analysis more transparent, probably more workers in the field are fluent in the tensor formalism, which on the other hand is indeed more straightforward for some of the practical applications. We have also (1) further clarified the geometric interpretations of the relevant versions of scale transformations and presented the systematical analysis of all of them in a unified framework. Concrete generalisations of the results in the previous literature are that we have (2) taken into account the possible couplings between torsion and non-metricity and (3) included the CP-violating terms. The map to the main results was given in Table 2, and furthermore, in Table 3, we present a summary of the invariants we have derived in Sections 4 and 5.

Table 3. The scalars and co-covariants in the three versions of rescalings, separated according to parity.

(The linearly independent combinations are not unique of course.) In the odd-parity cases, $n=4$.

\begin{tabular}{ccccc}
\hline Covariance & $Q^{2}$ & $T^{2}$ & $Q T$ & $\Sigma$ \\
\hline coordinate + & $A_{1}, A_{2}, A_{3}, A_{4}, A_{5}$ & $B_{1}, B_{2}, B_{3}$ & $C_{1}, C_{2}, C_{3}$ & 11 \\
coordinate - & $A_{6}$ & $B_{5}, B_{6}$ & $C_{4}, C_{5}, C_{6}$ & 6 \\
projective + & $\left(A_{1}-\frac{1}{n} A_{3}\right),\left(A_{2}-A_{4}\right),\left(A_{1}+\frac{1}{n} A_{2}-2 A_{5}\right),\left(B_{1}+2 B_{2}\right),\left(B_{1}-\frac{2}{n-1} B_{3}\right),\left(C_{1}-\frac{1}{2} C_{2}\right),\left(\frac{1}{8 n} A_{1}-\frac{1}{8} A_{2}+C_{1}-\frac{1}{n} C_{2}\right),\left(\frac{n-1}{8} A_{2}-B_{1}-C_{3}\right)$ & 8 \\
projective - & $A_{6}$ & $\left(B_{6}+4 C_{4}\right),\left(B_{6}+C_{5}\right),\left(B_{6}+C_{6}\right),\left(4 B_{5}+3 C_{6}\right)$ & 5 \\
conformal + & $\left(n A_{1}-A_{3}\right),\left(A_{2}-A_{4}\right),\left(A_{1}+\frac{1}{n} A_{2}-2 A_{5}\right)$ & $B_{1}, B_{2}, B_{3}$ & $\left(C_{1}-C_{3}\right),\left(n C_{1}-C_{2}\right)$ & 8 \\
conformal - & $A_{6}$ & $B_{5}, B_{6}$ & $\left(C_{4}-4 C_{5}\right),\left(C_{4}-4 C_{6}\right)$ & 5 \\
rescaling + & $A_{1}, A_{2}, A_{3}, A_{4}, A_{5}$ & $\left(B_{1}+2 B_{2}\right),\left(B_{2}+\frac{1}{n-1} B_{3}\right)$ & $(n-1) C_{1}-C_{2}+C_{3}$ & 8 \\
rescaling - & $A_{6}$ & $\left(4 B_{5}-3 B_{6}\right)$ & $C_{4}, C_{5}, C_{6}$ & 5 \\
\hline
\end{tabular}

Author Contributions: Conceptualization, methodology, validation, formal analysis, investigation, writing-original draft preparation, writing-review and editing: D.I. and T.K.; supervision, project administration, funding acquisition: T.K.

Funding: This work was supported by the Estonian Research Council grant PRG356 and the CoE grant TK133.

Acknowledgments: We would like to thank Ott Vilson for useful remarks.

Conflicts of Interest: The authors declare no conflict of interest.

\section{Abbreviations}

The following abbreviations are used in this manuscript:

GR General theory of Relativity

MAG Metric Affine Gauge theory 


\section{Appendix A. The Quadratic Non-Metricity and Torsion Scalars}

In this Appendix we derive the quadratic invariants of torsion and non-metricity. First we write systematically down all the possible contraction of the tensors Equations (8) and (9), and then sort out the independent ones. We end up with the same set of scalars as in e.g., Reference [71].

Let us thus first list the relevant scalars. The pure non-metricity scalars are

$$
\begin{gathered}
A_{1}=Q_{\alpha \mu \nu} Q^{\alpha \mu v} \\
A_{2}=Q_{\alpha \mu \nu} Q^{\mu v \alpha} \\
A_{3}=Q_{\mu} Q^{\mu} \\
A_{4}=q_{\mu} q^{\mu} \\
A_{5}=Q_{\mu} q^{\mu} \\
A_{6}=\epsilon^{\alpha \beta \gamma \delta} Q_{\alpha \beta \mu} Q_{\gamma \delta}{ }^{\mu}
\end{gathered}
$$

where the two independent traces are defined ${ }^{12}$ as $Q_{\alpha} \equiv Q_{\alpha \mu \nu} g^{\mu v}$ and $q_{\mu}=Q_{\lambda v \mu} g^{\lambda v}$. Please note that there appear to be five independent quadratic even invariants, though only four irreducible components of the the non-metricity tensor (the binom, conom, vecnom and conom, see the Appendix B.1 of Reference [7]).

The pure torsion scalars one may write down are

$$
\begin{gathered}
B_{1}=S_{\alpha \mu \nu} S^{\alpha \mu \nu} \\
B_{2}=S_{\alpha \mu \nu} S^{\mu v \alpha} \\
B_{3}=S_{\mu} S^{\mu} \\
B_{4}=t_{\mu} t^{\mu} \\
B_{5}=S_{\mu} t^{\mu} \\
B_{6}=\epsilon^{\alpha \beta \gamma \delta} S_{\alpha \beta \mu} S_{\gamma \delta}{ }^{\mu} \\
B_{7}=\epsilon^{\alpha \beta \gamma \delta} S_{\lambda \alpha \beta} S_{\gamma \delta}^{\lambda} \\
B_{8}=\epsilon^{\alpha \beta \gamma \delta} S_{\mu \alpha \beta} S_{\gamma \delta}{ }^{\mu}
\end{gathered}
$$

where $S_{\mu} \equiv S_{\mu \lambda}{ }^{\lambda}$ and $t^{\alpha} \equiv \epsilon^{\alpha \beta \gamma \delta} S_{\beta \gamma \delta}$. It should be noted that $B_{4}$ is not independent of the $B_{1}, B_{2}$ and $B_{3}$, and only two of the four pseudoscalars $B_{5}, B_{6}, B_{7}$ and $B_{8}$ is independent. Let first us show the redundancy of $B_{4}=t_{\mu} t^{\mu}$. By a direct calculation, this is found to be

$$
\begin{aligned}
B_{4} & =t_{\mu} t^{\mu}=\epsilon_{\mu \alpha \beta \gamma} \epsilon^{\mu \kappa \lambda \rho} S^{\alpha \beta \gamma} S_{\kappa \lambda \rho}=-3 ! \delta_{\alpha}^{[\kappa} \delta_{\beta}^{\lambda} \delta_{\gamma}^{\rho]} S^{\alpha \beta \gamma} S_{\kappa \lambda \rho}=-3 ! S^{\alpha \beta \gamma} S_{[\alpha \beta \gamma]} \\
& =-2 S^{\alpha \beta \gamma}\left(S_{\alpha \beta \gamma}+S_{\gamma \beta \alpha}+S_{\beta \gamma \alpha}\right)=-2\left(B_{1}+2 B_{2}\right) .
\end{aligned}
$$

Thus we may discard $B_{4}$ in the following without loss of generality. Let us then consider the parity-odd torsion scalars in $n=4$. We start from the definition of $t^{\rho}$, which when contracted by $\epsilon_{\rho \alpha \beta \mu}$ and using $\epsilon^{\rho \kappa \lambda \sigma} \epsilon_{\rho \alpha \beta \mu}=-3 ! \delta_{\alpha}^{[\kappa} \delta_{\beta}^{\lambda} \delta_{\mu}^{\sigma]}$ gives

$$
\epsilon_{\rho \alpha \beta \mu} t^{\rho}=-3 ! S_{[\alpha \beta \mu]} .
$$

Exploiting the antisymmetry of the torsion tensor in its first two indices the above may be expressed as

12 We are using $q_{\mu}=Q_{\alpha \mu \nu} g^{\alpha v}$ for the second non-metricity vector, instead of $\tilde{Q}_{\mu}[64,78]$ to avoid confusion that may appear from the various symbols. 


$$
\epsilon_{\rho \alpha \beta \mu} t^{\rho}=-2\left(S_{\alpha \beta \mu}+S_{\mu \alpha \beta}+S_{\beta \mu \alpha}\right) .
$$

Furthermore, contracting the above with $\epsilon^{\alpha \beta \gamma \delta}$ and using $\epsilon_{\rho \alpha \beta \mu} \epsilon^{\alpha \beta \gamma \delta}=-4 \delta_{\rho}^{[\gamma} \delta_{\mu}^{\delta]}$ we finally arrive at

$$
2 t^{[\gamma} \delta_{\mu}^{\delta]}=\epsilon^{\alpha \beta \gamma \delta} S_{\alpha \beta \mu}+2 \epsilon^{\alpha \beta \gamma \delta} S_{\mu \alpha \beta} .
$$

The latter is the key equation that gives the relations among the parity-odd terms. To obtain these, we first contract Equation (A6) by $S_{\gamma \delta}{ }^{\mu}$ and use the definitions of $B_{i}^{\prime} s$ to obtain

$$
2 B_{5}=B_{6}+2 B_{8}
$$

In addition, contracting with $S^{\mu}{ }_{\gamma \delta}$ this time, gives

$$
-B_{5}=B_{8}+2 B_{7}
$$

Therefore, we have two equations relating the $B_{5}, \ldots, B_{8}$ and so only two of the four are independent. We may choose the $B_{5}$ and $B_{6}$.

Finally, it is possible to form scalars by mixing non-metricity and torsion. Such invariants are

$$
\begin{gathered}
C_{1}=Q_{\alpha \mu \nu} S^{\alpha \mu \nu} \\
C_{2}=Q_{\mu} S^{\mu} \\
C_{3}=q_{\mu} S^{\mu} \\
C_{4}=Q^{\mu} t_{\mu} \\
C_{5}=q^{\mu} t_{\mu} \\
C_{6}=\epsilon^{\alpha \beta \gamma \delta} Q_{\alpha \beta \mu} S_{\gamma \delta}{ }^{\mu} \\
C_{7}=\epsilon^{\alpha \beta \gamma \delta} Q_{\alpha \beta \mu} S_{\gamma \delta}^{\mu}
\end{gathered}
$$

Again, there is redundancy in parity-odd terms. Out of the four combinations $C_{4}, C_{5}, C_{6}, C_{7}$ only the three are independent. This is easily seen by contracting Equation (A6) with $Q_{\gamma \delta}{ }^{\mu}$ to arrive at

$$
C_{4}-C_{5}=C_{6}+2 C_{7} \text {. }
$$

Therefore one scalar is redundant and we choose to disregard $C_{7}$.

This exhausts the list of the quadratic second order scalars in metric-affine geometry. Further reason, besides that their transformation properties are trivial, that we need not consider the curvature invariants beyond the $R=g^{\mu v} R^{\alpha}{ }_{\mu \alpha v}$ and the $\epsilon^{\alpha \beta \gamma \delta} R_{\alpha \beta \gamma \delta}$, is that by by decomposing the connection into the metric Equation (2), the non-metric part (known often as disformation) and the torsion part (known often as contortion) we can always rewrite all the curvature terms of the metric invariants and the above scalars (and their higher derivatives, which we leave out of from the present analysis). Note also that the linear scalars $\nabla_{\mu} T^{\mu}, \nabla_{\mu} Q^{\mu}$ and $\nabla_{\mu} q^{\mu}$ are redundant, up to boundary terms.

A remark about the parity-odd terms is in order. While our construction is the most general for any dimensions for the parity-even terms, it is stricly speaking restricted to $n=4$ when considering the parity-odd terms. The reason is that in $n$ dimensions one would have at hand the $n$-dimensional totally antisymmetric Levi-Civita symbol, which is a technical complication. Here we restrict to using the symbol only with four indices.

\section{Appendix A.1. On Quartic Invariants}

Having established the transformation laws for the quadratic torsion and non-metricity scalars in Section 4, we may us now find some (of the many!) quartic combinations that remain invariant, for example, under conformal metric transformations. To start with, let us first note that 


$$
\begin{aligned}
&\left(n \bar{A}_{1}-\bar{A}_{3}\right)=e^{-2 \phi}\left(n A_{1}-A_{3}\right) \\
&\left(\bar{A}_{2}-\bar{A}_{4}\right)=e^{-2 \phi}\left(A_{2}-A_{4}\right) \\
&\left(\bar{A}_{5}-\frac{n}{2} \bar{A}_{4}-\frac{1}{2 n} \bar{A}_{3}\right)=e^{-2 \phi}\left(A_{5}-\frac{n}{2} A_{4}-\frac{1}{2 n} A_{3}\right) \\
& \bar{B}_{i}=e^{-2 \phi} B_{i} \quad \forall i \\
&\left(\bar{C}_{1}-\bar{C}_{3}\right)=e^{-2 \phi}\left(C_{1}-C_{3}\right) \\
&\left(n \bar{C}_{1}-\bar{C}_{2}\right)=e^{-2 \phi}\left(n C_{1}-C_{2}\right) \\
&\left(n \bar{C}_{3}-\bar{C}_{2}\right)=e^{-2 \phi}\left(n C_{3}-C_{2}\right) \\
&\left(2 \bar{C}_{2}-n \bar{C}_{1}-n \bar{C}_{3}\right)=e^{-2 \phi}\left(2 C_{2}-n C_{1}-n C_{3}\right)
\end{aligned}
$$

under $\bar{g}_{\mu v}=e^{2 \phi} g_{\mu \nu}$. This in turn means that any of the above combinations when squared or multiplied by another combination of the list, yields a conformally invariant scalar. For instance, in $n=4$,

$$
\begin{aligned}
& \sqrt{-g}\left(4 A_{1}-A_{3}\right)^{2} \\
& \sqrt{-g}\left(A_{2}-A_{4}\right) B_{2}
\end{aligned}
$$

are both conformally invariant. In total we can form $7^{2}=49$ conformal invariants from the squares of the even-parity quadratic covariant combinations. However, the total number of conformal invariants is probably larger, since there are more scalars one can form by contracting the indices 4 tensors than the square of the number of scalars formed by contracting the indices of two tensors.

\section{Appendix B. Variational Identities}

In this Section, we will derive some preliminary results which will be helpful in the rest of this paper. First, we derive some necessary variational formulae. As our aim to to construct scale-invariant theories, we will elucidate the generic relations between three versions of scale-invariance and the tracelessness properties of the variational terms (without yet specifying the particular actions).

\section{Appendix B.1. Variations}

Let us gather here the various variations that we will use in what follows. We start with torsion and compute variations with respect to the metric first. We have

$$
T_{\mu \nu \lambda}\left(\delta_{g} S^{\mu \nu \lambda}\right)=\delta g^{\mu \nu}\left(T_{\mu \alpha \beta} S_{v}^{\alpha \beta}-T_{\alpha \nu \beta} S_{\mu}^{\alpha \beta}\right)=\delta g^{\mu \nu}\left(2 T_{[\nu \alpha] \beta} S_{\mu}^{\alpha \beta}\right)
$$

and also

$$
T^{\mu \nu \lambda}\left(\delta_{g} S_{\mu \nu \lambda}\right)=-\delta g^{\mu \nu}\left(T^{\alpha \beta}{ }_{\nu \beta \beta \mu}\right)
$$

where $T_{\mu \nu \lambda}$ is an arbitrary tensor field (or tensor density). Then setting $T_{\mu \nu \lambda}=S_{\mu \nu \lambda}$ one has

$$
S_{\mu \nu \lambda}\left(\delta_{g} S^{\mu \nu \lambda}\right)=\delta g^{\mu \nu}\left(2 S_{\nu \alpha \beta} S_{\mu}^{\alpha \beta}\right)
$$

as well as

$$
S^{\mu \nu \lambda}\left(\delta_{g} S_{\mu \nu \lambda}\right)=-\delta g^{\mu \nu}\left(S_{\alpha \beta \mu} S^{\alpha \beta}{ }_{v}\right)
$$

such that

$$
\delta_{g}\left(S_{\mu \nu \lambda} S^{\mu \nu \lambda}\right)=\delta g^{\mu \nu}\left(2 S_{\nu \alpha \beta} S_{\mu}^{\alpha \beta}-S_{\alpha \beta \mu} S^{\alpha \beta}{ }_{\nu}\right) .
$$

Therefore, setting $T_{\mu v \lambda}=S_{\lambda \mu v}$ we can conclude that

$$
\delta_{g}\left(S_{\mu \nu \lambda} S^{\lambda \mu \nu}\right)=-S_{v \alpha \beta} S_{\mu}^{\alpha \beta}\left(\delta g^{\mu \nu}\right) .
$$


Now, using

$$
\delta_{g} \epsilon_{\alpha \beta \gamma \delta}=\delta_{g}\left(\sqrt{-g} \eta_{\alpha \beta \gamma \delta}\right)=-\frac{1}{2} \epsilon_{\alpha \beta \gamma \delta} g_{\mu \nu} \delta g^{\mu \nu}
$$

we compute

$$
A^{\alpha} \delta_{g} t_{\alpha}=\delta g^{\mu \nu}\left[-\frac{1}{2} g_{\mu \nu} A_{\alpha} t^{\alpha}+2 A^{\lambda} \epsilon_{\lambda v \alpha \beta} S_{\mu}{ }^{\alpha \beta}\right]
$$

where $A^{\mu}$ is an arbitrary vector. Then, also using that $\delta_{g} S_{\mu}=0$ we find

$$
\delta_{g}\left(t_{\alpha} S^{\alpha}\right)=\delta g^{\mu \nu}\left[-\frac{1}{2} g_{\mu \nu} S_{\alpha} t^{\alpha}+2 S^{\lambda} \epsilon_{\lambda \nu \alpha \beta} S_{\mu}^{\alpha \beta}+t_{\mu} S_{\nu}\right]
$$

and also

$$
\delta_{g}\left(S_{\alpha} S^{\alpha}\right)=\delta g^{\mu \nu}\left(S_{\mu} S_{v}\right) .
$$

Following the same procedure for the rest of the quadratic torsion scalars, we finally derive the metric variations

$$
\begin{gathered}
\delta_{g} B_{1}=\delta g^{\mu \nu}\left(2 S_{\nu \alpha \beta} S_{\mu}^{\alpha \beta}-S_{\alpha \beta \mu} S^{\alpha \beta}{ }_{\nu}\right) \\
\delta_{g} B_{2}=\delta g^{\mu \nu}\left(-S_{v \alpha \beta} S_{\mu}{ }^{\alpha \beta}\right) \\
\delta_{g} B_{3}=\delta g^{\mu v}\left(S_{\mu} S_{v}\right) \\
\delta_{g} B_{5}=\delta g^{\mu \nu}\left[-\frac{1}{2} g_{\mu \nu} S_{\alpha} t^{\alpha}+2 S^{\lambda} \epsilon_{\lambda \nu \alpha \beta} S_{\mu}{ }^{\alpha \beta}+t_{\mu} S_{v}\right] \\
\delta_{g} B_{6}=\delta g^{\mu \nu}\left(\frac{1}{2} g_{\mu v} B_{6}-\epsilon^{\alpha \beta \gamma \delta} S_{\alpha \beta \mu} S_{\gamma \delta v}\right) \\
\delta_{g} B_{7}=\delta g^{\mu \nu}\left(\frac{1}{2} g_{\mu \nu} B_{7}+2 S^{\alpha \beta}{ }_{\mu} S_{\alpha}{ }^{\gamma \delta} \epsilon_{\nu \beta \gamma \delta}+\epsilon^{\alpha \beta \gamma \delta} S_{\mu \alpha \beta} S_{v \gamma \delta}\right) \\
\delta_{g} B_{8}=\delta g^{\mu v}\left(\frac{1}{2} g_{\mu \nu} B_{8}-\epsilon^{\beta}{ }_{\nu \gamma \delta} S^{\gamma \delta \alpha} S_{\mu \alpha \beta}\right) .
\end{gathered}
$$

Finally, we shall also need the variations of with respect to the connection. For the $\Gamma$-variations of non-metricity scalars we find

$$
\begin{gathered}
\delta_{\Gamma} A_{1}=\delta_{\Gamma}\left(Q_{\alpha \mu \nu} Q^{\alpha \mu \nu}\right)=\left(4 Q^{v \mu}{ }_{\lambda}\right) \delta \Gamma^{\lambda}{ }_{\mu \nu} \\
\delta_{\Gamma} A_{2}=\delta_{\Gamma}\left(Q_{\alpha \mu \nu} Q^{\mu v \alpha}\right)=2\left(Q^{\mu v}{ }_{\lambda}+Q_{\lambda}{ }^{\mu v}\right) \delta \Gamma^{\lambda}{ }_{\mu \nu} \\
\delta_{\Gamma} A_{3}=\delta_{\Gamma}\left(Q_{\mu} Q^{\mu}\right)=\left(4 Q^{v} \delta^{\mu}{ }_{\lambda}\right) \delta \Gamma^{\lambda}{ }_{\mu v} \\
\delta_{\Gamma} A_{4}=\delta_{\Gamma}\left(\tilde{Q}_{\mu} \tilde{Q}^{\mu}\right)=2\left(\tilde{Q}_{\lambda} g^{\mu v}+\tilde{Q}^{\mu} \delta_{\lambda}^{v}\right) \delta \Gamma^{\lambda}{ }_{\mu \nu} \\
\delta_{\Gamma} A_{5}=\delta_{\Gamma}\left(Q_{\mu} \tilde{Q}^{\mu}\right)=\left(2 \tilde{Q}^{v} \delta_{\lambda}^{\mu}+Q_{\lambda} g^{\mu v}+Q^{\mu} \delta_{\lambda}^{v}\right) \delta \Gamma_{\mu \nu}^{\lambda} .
\end{gathered}
$$

The $\Gamma$-variations of the torsion are straightforward. We are now armed with the formulas that allow to readily obtain the field equations for an arbitrary metric-affine theory consisting of the scalars in Appendix A.

\section{Appendix B.2. Projective Invariance and Tracelessness}

As we have already pointed out in the previous Section 3, the Palatini Tensor

$$
P_{\lambda}^{\mu v} \equiv \frac{\delta R}{\delta \Gamma^{\lambda}{ }_{\mu v}}
$$

has zero trace when contracted in its first two indices, that is

$$
P_{\mu}^{\mu \nu}=0 \text {. }
$$


In fact as we have argued before, any tensor constructed out of a projective invariant quantity has this property. Let us prove this here. Consider the scalar quantity $\Psi$ that is invariant under projective transformations. Then define

$$
\Xi_{\lambda} \mu \nu \equiv \frac{\delta \Psi}{\delta \Gamma^{\lambda} \mu \nu}
$$

Now consider the projective transformation $\Gamma_{\mu \nu}^{\lambda} \longrightarrow \hat{\Gamma}_{\mu v}^{\lambda}=\Gamma_{\mu \nu}^{\lambda}+\delta_{\mu}^{\lambda} \xi_{v}$ such that ${ }^{13}$

$$
\hat{\delta} \Gamma_{\mu v}^{\lambda}=\hat{\Gamma}_{\mu v}^{\lambda}-\Gamma_{\mu v}^{\lambda}=\delta_{\mu}^{\lambda} \xi_{v}
$$

Applying the latter transformation to $\psi$, we have

$$
\hat{\delta} \psi=\frac{\delta \psi}{\hat{\delta} \Gamma^{\lambda}{ }_{\mu \nu}} \hat{\delta} \Gamma_{\mu v}^{\lambda}=\Xi_{\lambda}^{\mu v} \delta_{\mu}^{\lambda} \xi_{v}=\Xi_{\mu}^{\mu v} \xi_{v}
$$

Now since $\psi$ is invariant, we have that $\hat{\delta} \psi=0$. Thus, using this, along with the fact that the vector $\xi_{v}$ is arbitrary, from the above we conclude that

$$
\Xi_{\mu}^{\mu \nu}=0
$$

as we had stated.

\section{Appendix B.3. Conformal Invariance and Tracelessness}

As we have proven above, if a scalar quantity is invariant under projective transformations then its variation with respect to the connection yields a tensor (or tensor density if we do not divide the result by $\sqrt{-g}$ ) that is traceless in its first two indices. Similarly, if a scalar density (which we may integrate to construct an action of course) is invariant under conformal transformations then its variation with respect to the metric tensor yields a tensor that is traceless. Let us prove this here.

Proof. Consider the scalar density $\sqrt{-g} \Psi$, where $\Psi$ is again a scalar. Then define the variation

$$
M_{\mu \nu} \equiv \frac{1}{\sqrt{-g}} \frac{\delta(\sqrt{-g} \Psi)}{\delta g^{\mu \nu}}
$$

and denote its trace by $M \equiv M_{\mu v} g^{\mu \nu}$. Consider now a conformal transformation of the metric $\bar{g}_{\mu v}=e^{2 \phi} g_{\mu v}$, or in its contravariant form $\bar{g}^{\mu v}=e^{-2 \phi} g^{\mu \nu}$. Expanding the latter for infinitesimal transformations, it follows that

$$
\bar{g}^{\mu \nu} \approx(1-2 \phi) g^{\mu \nu} \Rightarrow \bar{\delta} g^{\mu \nu}=-2 \phi g^{\mu \nu}
$$

where $\bar{\delta} g^{\mu v} \equiv \bar{g}^{\mu v}-g^{\mu v}$ denotes the infinitesimal change the metric undergoes under the conformal transformation. Given that $\sqrt{-g} \Psi$ is invariant under conformal transformations we have

$$
\bar{\delta}(\sqrt{-g} \Psi)=0 \Rightarrow M_{\mu \nu} \bar{\delta} g^{\mu \nu}=0 \Rightarrow-2 \phi M_{\mu \nu} g^{\mu \nu}=0
$$

and since the last one must hold true for arbitrary $\phi$ we conclude that

$$
M=M_{\mu \nu} g^{\mu \nu}=0
$$

as stated.

$13 \hat{\delta}$ denotes a projective variation of the connection, and in the following $\bar{\delta}$ will denote the variation under the conformal transformation and $\tilde{\delta}$ the variation under the frame rescaling. 
Example A1. Let us confirm the strength of the above statement with two examples. First consider the scalar density in 4 dimensions ${ }^{14}$

$$
\sqrt{-g} R^{2}
$$

which is conformally invariant as can be easily seen. Its metric variation is found to be

$$
M_{\mu \nu}=\frac{1}{\sqrt{-g}} \frac{\delta\left(\sqrt{-g} R^{2}\right)}{\delta g^{\mu \nu}}=2 R\left(R_{\mu \nu}-\frac{1}{4} g_{\mu \nu} R\right)
$$

and therefore

$$
M=M_{\mu \nu} g^{\mu \nu}=2 R(R-R)=0
$$

as expected. As a second example consider

$$
\sqrt{-g} R_{\mu v} R^{\mu v}
$$

which is also a conformally invariant quantity in 4 dimensions (as we recall, is any possible curvature-squared term). Variation with respect to the metric yields

$$
M_{\mu \nu}=\frac{1}{\sqrt{-g}} \frac{\delta\left(\sqrt{-g} R_{\mu v} R^{\mu \nu}\right)}{\delta g^{\mu \nu}}=-\frac{1}{2} R_{\alpha \beta} R^{\alpha \beta} g_{\mu \nu}+R_{\mu}{ }^{\beta} R_{\nu \beta}+R^{\beta}{ }_{\nu} R_{\beta \mu}
$$

which again gives a vanishing trace since

$$
M=M_{\mu \nu} g^{\mu \nu}=-2 R_{\mu \nu} R^{\mu \nu}+R_{\mu \nu} R^{\mu \nu}+R_{\mu \nu} R^{\mu v}=0 .
$$

Please note that from these considerations apply also for any matter source. It is indeed very well-known that the conformal symmetry is compatible only with traceless matter sources, the Maxwell field being the seminal example.

Appendix B.4. The Case of Frame Rescalings

As we have seen a frame rescaling results in a conformal transformation + a special projective transformation both powered by a single scalar field $\phi(x) .{ }^{15}$ We will now prove that if a scalar density is invariant under frame rescalings then the trace of its metric conjugate and the divergence of the trace of its connection conjugate are related to one another.

Proof. Consider the action

$$
S=\int \mathrm{d}^{n} x \sqrt{-g} \Psi
$$

and recall the definitions of the metric and connection conjugates

$$
M_{\mu \nu} \equiv \frac{1}{\sqrt{-g}} \frac{\delta(\sqrt{-g} \Psi)}{\delta g^{\mu \nu}}, \quad \Xi_{\lambda}^{\mu \nu} \equiv \frac{1}{\sqrt{-g}} \frac{\delta(\sqrt{-g} \mathcal{L})}{\delta \Gamma^{\lambda} \mu \nu}=\frac{\delta \mathcal{L}}{\delta \Gamma^{\lambda} \mu \nu}
$$

and the definitions of the traces

$$
M=g^{\mu v} M_{\mu v}, \quad \Xi^{\mu}=\Xi_{\lambda}^{\lambda \mu}
$$

We now state that if Equation (A38) is invariant under frame rescalings then

14 This of course generalizes to any dimension and takes the form $\sqrt{-g} R^{\frac{n}{2}}$ where $n$ is the dimension of the space.

15 This is most important because one can also have projective and conformal transformations that are powered by different fields. Then invariance means that both metric and connection conjugates have zero traces and they are not related. As an example consider $\sqrt{-g} R^{2}$ which is independently invariant under $\Gamma_{\mu \nu}^{\lambda} \rightarrow \Gamma_{\mu \nu}^{\lambda}+\delta_{\mu}^{\lambda} \xi_{v}$ and $g_{\mu \nu} \rightarrow e^{2 \phi} g_{\mu \nu}\left(\right.$ where $\xi_{v}$ and $\phi$ are not related to any way) and as a result $M=0$ and $\Xi^{\mu}=0$. 


$$
2 M+\frac{\partial_{\mu}\left(\sqrt{-g} \Xi^{\mu}\right)}{\sqrt{-g}}=0 .
$$

To prove this let us compute the change in Equation (A38) under frame rescalings. Using $\tilde{\delta} g^{\mu v}=\tilde{g}^{\mu \nu}-g^{\mu v}=-2 \phi g^{\mu v}$ and $\tilde{\delta} \Gamma_{\mu v}^{\lambda}=\tilde{\Gamma}_{\mu \nu}^{\lambda}-\Gamma_{\mu \nu}^{\lambda}=\delta_{\mu}^{\lambda} \partial_{\nu} \phi$ where $\tilde{\delta}$ denotes the change under frame rescalings, we compute

$$
\begin{aligned}
\tilde{\delta} S & =\int \mathrm{d}^{n} x\left[\sqrt{-g} M_{\mu v} \tilde{\delta} g^{\mu \nu}+\sqrt{-g} \Xi_{\lambda}{ }^{\mu v} \tilde{\delta}^{\lambda}{ }_{\mu v}\right]=\int \mathrm{d}^{n} x\left[\sqrt{-g}\left(-2 \phi g^{\mu \nu} M_{\mu v}\right)+\sqrt{-g} \Xi_{\lambda}{ }^{\mu v} \delta_{\mu}^{\lambda} \partial_{\nu} \phi\right] \\
& =\int \mathrm{d}^{n} x\left[-\sqrt{-g} 2 \phi M+\sqrt{-g} \Xi^{\mu} \partial_{\mu} \phi\right]=-\int \mathrm{d}^{n} x\left[\sqrt{-g} 2 \phi M+\phi\left(\partial_{\mu} \sqrt{-g} \Xi^{\mu}\right)\right]+\int \mathrm{d}^{n} x \partial_{\mu}\left(\sqrt{-g} \Xi^{\mu} \phi\right) \\
& =-\int \mathrm{d}^{n} x \phi\left[\sqrt{-g} 2 M+\left(\partial_{\mu} \sqrt{-g} \Xi^{\mu}\right)\right]+\text { a boundary term. }
\end{aligned}
$$

Then, ignoring surface terms, since $S$ is invariant it follows that

$$
\tilde{\delta} S \Rightarrow \phi\left[\sqrt{-g} 2 M+\left(\partial_{\mu} \sqrt{-g} \Xi^{\mu}\right)\right]=0
$$

and since the last equality must be true for arbitrary $\phi$ we conclude that

$$
2 M+\frac{\partial_{\mu}\left(\sqrt{-g} \Xi^{\mu}\right)}{\sqrt{-g}}=0
$$

as stated. Notice that if the above result is applied for the matter part of the action, for frame rescaling invariant matter the traces of the energy momentum and hyper-momentum tensors are related through

$$
2 T+\frac{\partial_{\mu}\left(\sqrt{-g} \Delta^{\mu}\right)}{\sqrt{-g}}=0
$$

where $T=T_{\mu \nu} g^{\mu v}$ and $\Delta^{v}=\Delta_{\mu}^{\mu v}=0$.

\section{Appendix C. Transformation Identities}

In this Section we will investigate the transformation properties of the torsion tensor, nonmetricity tensor and the related quantities under the three versions of rescalings.

\section{Appendix C.1. Projective Transformations}

Recall that the projective transformation is $\Gamma^{\lambda}{ }_{\mu \nu} \rightarrow \hat{\Gamma}_{\mu v}^{\lambda}=\Gamma_{\mu v}^{\lambda}+\delta_{\mu}^{\lambda} \xi_{v}, g_{\mu v} \rightarrow \hat{g}_{\mu v}=g_{\mu v}$. It is easy to see that under the above, the torsion and non-metricity tensors transform as

$$
\hat{S}_{\mu \nu}^{\lambda}=S_{\mu \nu}^{\lambda}+\delta_{[\mu}^{\lambda} \xi_{v]} \quad \text { or } \quad \hat{S}_{\mu \nu \alpha}=S_{\mu \nu \alpha}+g_{\alpha[\mu} \xi_{v]}
$$

and

$$
\hat{Q}_{\alpha \mu \nu}=Q_{\alpha \mu \nu}+2 \xi_{\alpha} g_{\mu \nu} .
$$

For their associate vectors we have

$$
\hat{S}_{\mu}=S_{\mu}+\frac{(1-n)}{2} \xi_{\mu}, \quad \hat{Q}_{\mu}=Q_{\mu}+2 n \xi_{\mu}, \quad \text { and } \quad \hat{q}_{\mu}=q_{\mu}+2 \xi_{\mu} .
$$

Please note that this implies

$$
S_{[\mu v \alpha]} \longrightarrow S_{[\mu v \alpha]}
$$

the projective invariance of the totally antisymmetric torsion. It is this part of torsion that couples to fermions. Thus, the Dirac action is projectively invariant, in addition to being invariant under the frame rescalings. 


\section{Appendix C.2. Conformal Transformations}

What we call the conformal transformation in this paper is defined by $g_{\mu \nu} \rightarrow \bar{g}_{\mu v}=e^{2 \phi} g_{\mu \nu}$ and $\Gamma^{\lambda}{ }_{\mu \nu} \rightarrow \bar{\Gamma}^{\lambda}{ }_{\mu \nu}=\Gamma^{\lambda}{ }_{\mu v}$. That is, under a conformal transformation the metric tensor picks up a conformal factor $e^{2 \phi}$ while the affine connection is left unchanged. Please note that the contravariant form of the metric tensor transforms as $\bar{g}^{\mu \nu}=e^{-2 \phi} g^{\mu \nu}$ as can be easily seen from the relation $g_{\mu \nu} g^{v \lambda}=\delta_{\mu}^{\lambda}$. In addition, the square root of the determinant of the metric obeys the transformation rule

$$
\sqrt{-\bar{g}}=e^{n \phi} \sqrt{-g}
$$

and for $n=4$ thus $\sqrt{-\bar{g}}=e^{4 \phi} \sqrt{-g}$, which is obtained directly by first taking the determinant of $\bar{g}_{\mu v}$ and then taking the square root of the result. From this last relation we infer the transformation rule for the Levi-Civita tensor

$$
\begin{gathered}
\bar{\epsilon}_{\mu v \rho \sigma}=e^{4 \phi} \epsilon_{\mu v \rho \sigma} \\
\bar{\epsilon}^{\mu v \rho \sigma}=e^{-4 \phi} \epsilon^{\mu v \rho \sigma}
\end{gathered}
$$

and recall that $\epsilon_{\mu v \rho \sigma}=\sqrt{-g} \eta_{\mu v \rho \sigma}$ where $\eta_{\mu v \rho \sigma}$ is the Levi-Civita symbol. Using the above we see that torsion and non-metricity transform as

$$
\begin{gathered}
\bar{S}_{\mu \nu}{ }^{\lambda}=S_{\mu \nu}{ }^{\lambda} \\
\bar{Q}_{\alpha \mu \nu}=e^{2 \phi}\left(Q_{\alpha \mu \nu}-2\left(\partial_{\alpha} \phi\right) g_{\mu \nu}\right)
\end{gathered}
$$

and the related vectors as

$$
\bar{Q}_{\mu}=Q_{\mu}-2 n \partial_{\mu} \phi, \quad \bar{q}_{\mu}=q_{\mu}-2 \partial_{\mu} \phi
$$

and

$$
\bar{S}_{\mu}=S_{\mu}, \quad \bar{t}_{\mu}=t_{\mu} .
$$

Then, it follows that all pure torsion scalars

$$
B_{1}=S_{\alpha \mu \nu} S^{\alpha \mu \nu}, B_{2}=S_{\alpha \mu \nu} S^{\mu v \alpha}, B_{3}=S_{\mu} S^{\mu}, B_{4}=t_{\mu} t^{\mu}
$$

and

$$
B_{5}=S_{\mu} t^{\mu}, B_{6}=\epsilon^{\alpha \beta \gamma \delta} S_{\alpha \beta \mu} S_{\gamma \delta}{ }^{\mu}, B_{7}=\epsilon^{\alpha \beta \gamma \delta} S_{\lambda \alpha \beta} S_{\gamma \delta}^{\lambda}, B_{8}=\epsilon^{\alpha \beta \gamma \delta} S_{\mu \alpha \beta} S_{\gamma \delta}{ }^{\mu}
$$

are all conformally covariant, that is

$$
\bar{B}_{i}=e^{-2 \phi} B_{i}
$$

for any $i=1,2, \ldots, 8$. This means that any combination $\sqrt{-g} B_{i} B_{j}$ is conformal invariant when $n=4$. Regarding the pure non-metricity scalars, one can verify the transformation laws

$$
\begin{gathered}
\bar{A}_{1}=\bar{Q}_{\alpha \mu \nu} \bar{Q}^{\alpha \mu \nu}=e^{-2 \phi}\left[A_{1}-4 Q^{\mu} \partial_{\mu} \phi+4 n(\partial \phi)^{2}\right] \\
\bar{A}_{2}=\bar{Q}_{\alpha \mu \nu} \bar{Q}^{\mu v \alpha}=e^{-2 \phi}\left[A_{2}-4 q^{\mu} \partial_{\mu} \phi+4(\partial \phi)^{2}\right] \\
\bar{A}_{3}=\bar{Q}_{\mu} \bar{Q}^{\mu}=e^{-2 \phi}\left[A_{3}-4 n Q^{\mu} \partial_{\mu} \phi+4 n^{2}(\partial \phi)^{2}\right] \\
\bar{A}_{4}=\bar{q}_{\mu} \bar{q}^{\mu}=e^{-2 \phi}\left[A_{4}-4 q^{\mu} \partial_{\mu} \phi+4(\partial \phi)^{2}\right] \\
\bar{A}_{5}=\bar{Q}_{\mu} \bar{q}^{\mu}=e^{-2 \phi}\left[A_{5}-2\left(Q^{\mu}+n q^{\mu}\right) \partial_{\mu} \phi+4 n(\partial \phi)^{2}\right] \\
\bar{A}_{6}=\bar{\epsilon}^{\alpha \beta \gamma \delta} \bar{Q}_{\alpha \beta \mu} \bar{Q}_{\gamma \delta}^{\mu}=e^{-2 \phi} A_{6}
\end{gathered}
$$


and for the mixed terms

$$
\begin{gathered}
\bar{C}_{1}=\bar{Q}_{\alpha \mu \nu} \bar{S}^{\alpha \mu \nu}=e^{-2 \phi}\left[C_{1}-2 S^{\mu} \partial_{\mu} \phi\right] \\
\bar{C}_{2}=\bar{Q}_{\mu} \bar{S}^{\mu}=e^{-2 \phi}\left[C_{2}-2 n S^{\mu} \partial_{\mu} \phi\right] \\
\bar{C}_{3}=\bar{q}_{\mu} \bar{S}^{\mu}=e^{-2 \phi}\left[C_{3}-2 S^{\mu} \partial_{\mu} \phi\right] \\
\bar{C}_{4}=\bar{Q}^{\mu} \bar{t}_{\mu}=e^{-2 \phi}\left[C_{4}-2 n t^{\mu} \partial_{\mu} \phi\right] \\
\bar{C}_{5}=\bar{q}^{\mu} \bar{t}_{\mu}=e^{-2 \phi}\left[C_{5}-2 t^{\mu} \partial_{\mu} \phi\right] \\
\tilde{C}_{6}=\bar{\epsilon}^{\alpha \beta \gamma \delta} \bar{Q}_{\alpha \beta \mu} \bar{S}_{\gamma \delta}{ }^{\mu}=e^{-2 \phi}\left[C_{6}-2 t^{\mu} \partial_{\mu} \phi\right] \\
\bar{C}_{7}=\bar{\epsilon}^{\alpha \beta \gamma \delta} \bar{Q}_{\alpha \beta \mu} \bar{S}_{\gamma \delta}^{\mu}=e^{-2 \phi}\left[C_{7}-2 t^{\mu} \partial_{\mu} \phi\right] .
\end{gathered}
$$

\section{Appendix C.3. Frame Rescaling}

A frame rescaling transformation results in a combination of a conformal metric transformation + a special projective transformation. More specifically, we have $\Gamma_{\mu \nu}^{\lambda} \rightarrow \tilde{\Gamma}_{\mu \nu}^{\lambda}=\Gamma_{\mu \nu}^{\lambda}+\delta_{\mu}^{\lambda} \partial_{\nu} \phi$ and $g_{\mu v} \rightarrow \tilde{g}_{\mu \nu}=e^{2 \phi} g_{\mu \nu}$, with the same scalar field $\phi(x)$ appearing in both above. Interestingly, under the this transformation, the non-metricity tensor does not change and it just picks-up a conformal factor. In words

$$
\tilde{Q}_{\alpha \mu \nu}=e^{2 \phi} Q_{\alpha \mu \nu}
$$

as can be easily seen by applying both transformations on Equation (9). This makes the procedure of computing quadratic non-metricity scalars, extremely simple. Indeed, let us recall the scalars

$$
A_{1}=Q_{\alpha \mu \nu} Q^{\alpha \mu v}, A_{2}=Q_{\alpha \mu \nu} Q^{\mu v \alpha}, A_{3}=Q_{\mu} Q^{\mu}, A_{4}=q_{\mu} q^{\mu}, A_{5}=Q_{\mu} q^{\mu}, A_{6}=\epsilon^{\alpha \beta \gamma \delta} Q_{\alpha \beta \mu} Q_{\gamma \delta}{ }^{\mu} .
$$

It is an easy matter to show that under frame rescalings

$$
\tilde{A}_{i}=e^{-2 \phi} A_{i}
$$

for any $i=1,2, \ldots, 6$. Therefore any combination $\sqrt{-g} A_{i} A_{j}$ is invariant under frame rescalings. As far as torsion is concerned, we have the transformation law

$$
\tilde{S}_{\mu \nu}^{\lambda}=S_{\mu \nu}^{\lambda}+\delta_{[\mu}^{\lambda} \partial_{\nu]} \phi
$$

and for the torsion vector

$$
\tilde{S}_{\mu}=S_{\mu}+\frac{(1-n)}{2} \partial_{\mu} \phi .
$$

Then, considering the even-parity torsion scalars Equation (A57) we see that under a frame rescaling, they transform as

$$
\begin{array}{r}
\tilde{B}_{1}=e^{-2 \phi}\left[B_{1}-2 S^{\mu} \partial_{\mu} \phi+\frac{(n-1)}{2}(\partial \phi)^{2}\right] \\
\tilde{B}_{2}=e^{-2 \phi}\left[B_{2}+S^{\mu} \partial_{\mu} \phi+\frac{(1-n)}{4}(\partial \phi)^{2}\right] \\
\tilde{B}_{3}=e^{-2 \phi}\left[B_{3}+(1-n) S^{\mu} \partial_{\mu} \phi+\frac{(1-n)^{2}}{4}(\partial \phi)^{2}\right]
\end{array}
$$

Notice that the combinations $B_{1}+2 B_{2},(n-1) B_{1}-2 B_{3}$ and $(n-1) B_{2}+B_{3}$ transform covariantly. For the odd-parity quadratic torsion scalars Equation (A58) one finds 


$$
\begin{gathered}
\tilde{B}_{4}=e^{-2 \phi} B_{4} \\
\tilde{B}_{5}=e^{-2 \phi}\left[B_{5}+\frac{(1-n)}{2} t^{\mu} \partial_{\mu} \phi\right] \\
\tilde{B}_{6}=e^{-2 \phi}\left[B_{6}-2 t^{\mu} \partial_{\mu} \phi\right] \\
\tilde{B}_{7}=e^{-2 \phi}\left[B_{7}+t^{\mu} \partial_{\mu} \phi\right] \\
\tilde{B}_{8}=e^{-2 \phi}\left[B_{8}-\frac{1}{2} t^{\mu} \partial_{\mu} \phi\right]
\end{gathered}
$$

For the mixed terms one finds

$$
\begin{gathered}
\tilde{C}_{1}=\tilde{Q}_{\alpha \mu \nu} \tilde{S}^{\alpha \mu \nu}=e^{-2 \phi}\left[C_{1}+\frac{1}{2}\left(q^{\mu}-Q^{\mu}\right) \partial_{\mu} \phi\right] \\
\tilde{C}_{2}=\tilde{Q}_{\mu} \tilde{S}^{\mu}=e^{-2 \phi}\left[C_{2}+\frac{1}{2}(1-n) Q^{\mu} \partial_{\mu} \phi\right] \\
\tilde{C}_{3}=\tilde{q}_{\mu} \tilde{S}^{\mu}=e^{-2 \phi}\left[C_{3}+\frac{1}{2}(1-n) q^{\mu} \partial_{\mu} \phi\right] \\
\tilde{C}_{4}=\tilde{Q}_{\mu} \tilde{t}^{\mu}=e^{-2 \phi} C_{4} \\
\tilde{C}_{5}=\tilde{q}_{\mu} \tilde{t}^{\mu}=e^{-2 \phi} C_{5} \\
\tilde{C}_{6}=\tilde{\epsilon}^{\alpha \beta \gamma \delta} \tilde{Q}_{\alpha \beta \mu} \tilde{S}_{\gamma \delta}{ }^{\mu}=e^{-2 \phi} C_{6} \\
\tilde{C}_{7}=\tilde{\epsilon}^{\alpha \beta \gamma \delta} \tilde{Q}_{\alpha \beta \mu} \tilde{S}_{\gamma \delta}^{\mu}=e^{-2 \phi} C_{6}
\end{gathered}
$$

Example A2. As an example consider the scalar density

$$
\sqrt{-g} A^{2}=\sqrt{-g}\left(Q_{\mu} Q^{\mu}\right)^{2}
$$

which is invariant under frame rescalings in $n=4$ as can be easily seen. Its metric conjugate reads (where we have dropped a total derivative that is assumed to vanish on the boundary)

$$
M_{\mu v}=-\frac{1}{2} g_{\mu v} A^{2}+2 A Q_{\mu} Q_{v}-4 g_{\mu \nu} \frac{\partial_{\alpha}\left(\sqrt{-g} Q^{\alpha} A\right)}{\sqrt{-g}}
$$

with the trace

$$
M=-16 \frac{\partial_{\alpha}\left(\sqrt{-g} Q^{\alpha} A\right)}{\sqrt{-g}}
$$

The associated connection conjugate is found to be

$$
\Xi_{\lambda}{ }^{\mu v}=8 A Q^{v} \delta_{\lambda}^{\mu}
$$

with the trace (in the first two indices)

$$
\Xi^{v}=32 A Q^{v}
$$

So we observe that

$$
\frac{\partial_{\mu}\left(\sqrt{-g} \Xi^{\mu}\right)}{\sqrt{-g}}=32 \frac{\partial_{\alpha}\left(\sqrt{-g} Q^{\alpha} A\right)}{\sqrt{-g}}=-2 M \quad \Rightarrow \quad 2 M+\frac{\partial_{\mu}\left(\sqrt{-g} \Xi^{\mu}\right)}{\sqrt{-g}}=0
$$

as expected.

\section{References}

1. Weyl, H. Gravitation and electricity. Sitzungsber. Preuss. Akad. Wiss. 1918, 26, 465-480. [CrossRef]

2. Weyl, H. Electron and gravitation. Z. Phys. 1929, 56, 330-352. (In German) [CrossRef]

3. O'Raifeartaigh, L. The Dawning of Gauge Theory; Princeton University Press: Princeton, NJ, USA, 1997. 
4. Koivisto, T. An integrable geometrical foundation of gravity. arXiv 2018, arXiv:1802.006502018.

5. Blagojević, M.; Hehl, F.W. (Eds.) Gauge Theories of Gravitation; World Scientific: Singapore, 2013.

6. Hooft, T.G. Local conformal symmetry: The missing symmetry component for space and time. Int. J. Mod. Phys. D 2015, 24, 1543001. [CrossRef]

7. Hehl, F.W.; McCrea, J.D.; Mielke, E.W.; Ne'eman, Y. Metric affine gauge theory of gravity: Field equations, Noether identities, world spinors, and breaking of dilation invariance. Phys. Rept. 1995, 258, 1-171. [CrossRef]

8. Mannheim, P.D. Making the case for conformal gravity. Found. Phys. 2012, 42, 388-420. [CrossRef]

9. Scholz, E. Weyl geometry in late 20th century physics. arXiv 2011, arXiv:1111.3220.

10. Nakayama, Y. Scale invariance vs. conformal invariance. Phys. Rept. 2015, 569, 1-93. [CrossRef]

11. Rachwał, L. Conformal symmetry in field theory and in quantum gravity. Universe 2018, 4, 125. [CrossRef]

12. Olmo, G.J. Palatini approach to modified gravity: $\mathrm{f}(\mathrm{R})$ theories and beyond. Int. J. Mod. Phys. D 2011, 20, 413-462. [CrossRef]

13. Heisenberg, L. A systematic approach to generalisations of General Relativity and their cosmological implications. arXiv 2018, arXiv:1807.01725.

14. Shapiro, I.L. Physical aspects of the space-time torsion. Phys. Rept. 2002, 357, 113. [CrossRef]

15. Hehl, F.W.; Obukhov, Y.N. Elie Cartan's torsion in geometry and in field theory, an essay. Annales Fond. Broglie 2007, 32, 157-194.

16. Vitagliano, V. The role of nonmetricity in metric-affine theories of gravity. Class. Quantum Gravity 2014, 31, 045006. [CrossRef]

17. Weyl, H. A remark on the coupling of gravitation and electron. Phys. Rev. 1950, 77, 699-701. [CrossRef]

18. Goenner, H.F.M. On the history of unified field theories. Part II. (ca. 1930-ca. 1965). Living Rev. Rel. 2014, 17, 5. [CrossRef]

19. Ehlers, J.; Pirani, F.A.E.; Schild, A. Republication of: The geometry of free fall and light propagation. Gen. Relat. Gravitity 2012, 44, 1587-1609. [CrossRef]

20. Magnano, G.; Sokolowski, L.M. On physical equivalence between nonlinear gravity theories and a general relativistic selfgravitating scalar field. Phys. Rev. D 1994, 50, 5039-5059. [CrossRef]

21. Faraoni, V.; Gunzig, E.; Nardone, P. Conformal transformations in classical gravitational theories and in cosmology. Fund. Cosmic Phys. 1999, 20, 121.

22. Allemandi, G.; Capone, M.; Capozziello, S.; Francaviglia, M. Conformal aspects of Palatini approach in extended theories of gravity. Gen. Rel. Gravity 2006, 38, 33-60. [CrossRef]

23. Iosifidis, D.; Petkou, A.C.; Tsagas, C.G. Torsion/non-metricity duality in $\mathrm{f}(\mathrm{R})$ gravity. arXiv 2018, arXiv:1810.06602.

24. Amendola, L.; Enqvist, K.; Koivisto, T. Unifying Einstein and Palatini gravities. Phys. Rev. D 2011, 83, 044016. [CrossRef]

25. Capozziello, S.; Harko, T.; Koivisto, T.S.; Lobo, F.S.N.; Olmo, G.J. Hybrid metric-Palatini gravity. Universe 2015, 1, 199-238. [CrossRef]

26. Beltran Jimenez, J.; Heisenberg, L.; Koivisto, T.S. Cosmology for quadratic gravity in generalized Weyl geometry. J. Cosmol. Astropart. Phys. 2016, 2016, 46. [CrossRef]

27. Iosifidis, D.; Tsagas, C.G.; Petkou, A.C. The Raychaudhuri equation in spacetimes with torsion and non-metricity. arXiv 2018, arXiv:1809.04992.

28. Shaposhnikov, M.; Zenhausern, D. Quantum scale invariance, cosmological constant and hierarchy problem. Phys. Lett. B 2009, 671, 162-166. [CrossRef]

29. Kannike, K.; Hütsi, G.; Pizza, L.; Racioppi, A.; Raidal, M.; Salvio, A.; Strumia, A. Dynamically induced Planck scale and inflation. J. High Energy Phys. 2015, 2015, 65. [CrossRef]

30. De Martini, F. The Higgs field and the resolution of the Cosmological Constant Paradox in the Weyl-geometrical Universe. Philos. Trans. R. Soc. Lond. A 2017, 375, 388. [CrossRef]

31. Lucat, S.; Prokopec, T.; Swiezewska, B. Conformal symmetry and the cosmological constant problem. arXiv 2018, arXiv:1804.00926.

32. Bamba, K.; Odintsov, S.D.; Sáez-Gómez, D. Conformal symmetry and accelerating cosmology in teleparallel gravity. Phys. Rev. D 2013, 88, 084042. [CrossRef]

33. Momeni, D.; Myrzakulov, R. Conformal invariant teleparallel cosmology. Eur. Phys. J. Plus 2014, $129,137$. [CrossRef] 
34. Silva, J.G.; Santos, A.F.; Ulhoa, S.C. On Friedmann-Robertson-Walker model in conformal teleparallel gravity. Eur. Phys. J. C 2016, 76, 167. [CrossRef]

35. Israelit, M. Quintessence and dark matter created by Weyl-Dirac geometry. Found. Phys. 2002, 32, 945-961. [CrossRef]

36. Lewandowski, A.; Meissner, K.A.; Nicolai, H. Conformal standard model, leptogenesis and dark matter. Phys. Rev. D 2018, 97, 035024. [CrossRef]

37. Lobo, F.S.N. General class of wormhole geometries in conformal Weyl gravity. Class. Quantum Gravity 2008, 25, 175006. [CrossRef]

38. Hohmann, M.; Pfeifer, C.; Raidal, M.; Veermäe, H. Wormholes in conformal gravity. J. Cosmol. Astropart. Phys. 2018, 2018, 3. [CrossRef]

39. Modesto, L.; Rachwal, L. Finite conformal quantum gravity and nonsingular spacetimes. arXiv 2016, arXiv:1605.04173.

40. Bambi, C.; Modesto, L.; Rachwał, L. Spacetime completeness of non-singular black holes in conformal gravity. J. Cosmol. Astropart. Phys. 2017, 2017, 3. [CrossRef]

41. Bambi, C.; Modesto, L.; Porey, S.; Rachwał, L. Black hole evaporation in conformal gravity. J. Cosmol. Astropart. Phys. 2017, 2017, 33. [CrossRef]

42. Bambi, C.; Modesto, L.; Porey, S.; Rachwał, L. Formation and evaporation of an electrically charged black hole in conformal gravity. Eur. Phys. J. C 2018, 78, 116. [CrossRef]

43. Hazboun, J.S.; Wheeler, J.T. Time and dark matter from the conformal symmetries of Euclidean space. Class. Quantum Gravity 2014, 31, 215001. [CrossRef]

44. Bezrukov, F.; Karananas, G.K.; Rubio, J.; Shaposhnikov, M. Higgs-Dilaton cosmology: An effective field theory approach. Phys. Rev. D 2013, 87, 096001. [CrossRef]

45. Ferreira, P.G.; Hill, C.T.; Ross, G.G. Weyl current, scale-invariant inflation and Planck scale generation. Phys. Rev. D 2017, 95, 043507. [CrossRef]

46. Gegenberg, J.; Rahmati, S.; Seahra, S.S. Big bounce, slow-roll inflation, and dark energy from conformal gravity. Phys. Rev. D 2017, 95, 043509. [CrossRef]

47. Bars, I.; Steinhardt, P.; Turok, N. Local conformal symmetry in physics and cosmology. Phys. Rev. D 2014, 89, 043515. [CrossRef]

48. Obukhov, Y.N. Conformal invariance and space-time torsion. Phys. Lett. A 1982, 90, 13-16. [CrossRef]

49. Zhytnikov, V.V. Conformally invariant Lagrangians in metric affine and Riemann-Cartan spaces. Int. J. Mod. Phys. A 1993, 8, 5141-5152. [CrossRef]

50. Shtanov, Y.V.; Yushchenko, S.A. Conformally invariant cosmology based on Riemann-Cartan space-time. Class. Quantum Gravity 1994, 11, 2455-2482. [CrossRef]

51. Karahan, C.N.; Dogangun, O.; Demir, D.A. Conformal transformations in metric-affine gravity and ghosts. Annalen Phys. 2012, 524, 461-469. [CrossRef]

52. Moon, T.Y.; Lee, J.; Oh, P. Conformal invariance in Einstein-Cartan-Weyl space. Mod. Phys. Lett. A 2010, 25, 3129-3143. [CrossRef]

53. Vazirian, R.; Tanhayi, M.R.; Motahar, Z.A. Weyl-invariant extension of the Metric-Affine Gravity. Adv. High Energy Phys. 2015, 2015, 902396. [CrossRef]

54. Wright, M. Conformal transformations in modified teleparallel theories of gravity revisited. Phys. Rev. D 2016, 93, 103002. [CrossRef]

55. Lucat, S.; Prokopec, T. The role of conformal symmetry in gravity and the standard model. Class. Quantum Gravity 2016, 33, 245002. [CrossRef]

56. Barnaveli, A.; Lucat, S.; Prokopec, T. Inflation as a spontaneous symmetry breaking of Weyl symmetry. arXiv 2018, arXiv:1809.10586.

57. Afonso, V.I.; Bejarano, C.; Beltran Jimenez, J.; Olmo, G.J.; Orazi, E. The trivial role of torsion in projective invariant theories of gravity with non-minimally coupled matter fields. Class. Quantum Gravity 2017, 34, 235003. [CrossRef]

58. Aoki, K.; Shimada, K. Galileon and generalized Galileon with projective invariance in a metric-affine formalism. Phys. Rev. D 2018, 98, 044038. [CrossRef]

59. Janssen, B.; Jimenez-Cano, A. Projective symmetries and induced electromagnetism in metric-affine gravity. arXiv 2018, arXiv:1807.10168.

60. Dirac, P.A.M. Long range forces and broken symmetries. Proc. R. Soc. Lond. A 1973, 333, 403-418. [CrossRef] 
61. Israelit, M. The Weyl-Dirac Theory and Our Universe; Nova Science Publishers: Hauppauge, NY, USA, 1999.

62. Faraoni, V.; Nadeau, S. The (pseudo)issue of the conformal frame revisited. Phys. Rev. D 2007, 75, 023501. [CrossRef]

63. Brans, C.; Dicke, R.H. Mach's principle and a relativistic theory of gravitation. Phys. Rev. 1961, 124, 925-935. [CrossRef]

64. Beltrán Jiménez, J.; Heisenberg, L.; Koivisto, T.S. Teleparallel Palatini theories. J. Cosmol. Astropart. Phys. 2018, 2018, 39. [CrossRef]

65. Beltran Jimenez, J.; Koivisto, T.S. Extended Gauss-Bonnet gravities in Weyl geometry. Class. Quantum Gravity 2014, 31, 135002. [CrossRef]

66. Beltran Jimenez, J.; Koivisto, T.S. Spacetimes with vector distortion: Inflation from generalised Weyl geometry. Phys. Lett. B 2016, 756, 400-404. [CrossRef]

67. Aringazin, A.K.; Mikhailov, A.L. Matter fields in spacetime with vector nonmetricity. Class. Quantum Gravity 1991, 8, 1685. [CrossRef]

68. Haghani, Z.; Khosravi, N.; Shahidi, S. The Weyl-Cartan Gauss-Bonnet gravity. Class. Quantum Gravity 2015, 32, 215016. [CrossRef]

69. Shahidi, S. Axionic extension of the Proca action. Phys. Rev. D 2018, 98, 084004. [CrossRef]

70. Beltrán Jiménez, J.; Koivisto, T.S. Modified gravity with vector distortion and cosmological applications. Universe 2017, 3, 47. [CrossRef]

71. Pagani, C.; Percacci, R. Quantum gravity with torsion and non-metricity. Class. Quantum Gravity 2015, 32, 195019. [CrossRef]

72. Jiménez, J.B.; Heisenberg, L.; Koivisto, T. Coincident general relativity. Phys. Rev. D 2018, 98, 044048. [CrossRef]

73. Nester, J.M.; Yo, H.J. Symmetric teleparallel general relativity. Chin. J. Phys. 1999, 37, 113.

74. Adak, M.; Sert, O.; Kalay, M.; Sari, M. Symmetric teleparallel gravity: Some exact solutions and spinor couplings. Int. J. Mod. Phys. A 2013, 28, 1350167. [CrossRef]

75. Rünkla, M.; Vilson, O. Family of scalar-nonmetricity theories of gravity. Phys. Rev. D 2018, 98, 084034. [CrossRef]

76. Golovnev, A.; Koivisto, T.; Sandstad, M. On the covariance of teleparallel gravity theories. Class. Quantum Gravity 2017, 34, 145013. [CrossRef]

77. Conroy, A.; Koivisto, T. The spectrum of symmetric teleparallel gravity. arXiv 2017, arXiv:1710.05708.

78. Järv, L.; Rünkla, M.; Saal, M.; Vilson, O. Nonmetricity formulation of general relativity and its scalar-tensor extension. Phys. Rev. D 2018, 97, 124025. [CrossRef]

79. Hohmann, M. Polarization of gravitational waves in general teleparallel theories of gravity. In Proceedings of the 3rd Zeldovich Meeting: An International Conference in Honor of Ya. B. Zeldovich, Minsk, Belarus, 23-27 April 2018.

80. Hohmann, M.; Pfeifer, C.; Said, J.L.; Ualikhanova, U. Propagation of gravitational waves in symmetric teleparallel gravity theories. arXiv 2018, arXiv:1808.02894.

81. Soudi, I.; Farrugia, G.; Gakis, V.; Said, J.L.; Saridakis, E.N. Polarization of gravitational waves in symmetric teleparallel theories of gravity and their modifications. arXiv 2018, arXiv:1810.08220.

82. Adak, M. Gauge Approach to the symmetric teleparallel gravity. arXiv 2018, arXiv:1809.01385. [CrossRef]

(C) 2019 by the authors. Licensee MDPI, Basel, Switzerland. This article is an open access article distributed under the terms and conditions of the Creative Commons Attribution (CC BY) license (http:/ / creativecommons.org/licenses/by/4.0/). 